\title{
Determining Timing Parameters for the Code Generation from Platform-Independent Timed Models
}

\author{
BAEKGYU KIM, Toyota InfoTechnology Center, USA \\ LU FENG, University of Virginia \\ OLEG SOKOLSKY and INSUP LEE, University of Pennsylvania
}

\begin{abstract}
Safety-critical embedded systems often need to meet dependability requirements such as strict input/output timing constraints. To meet the timing requirements, the code generation (e.g., C code) from timed models needs to determine the timing parameters that indicate when the code has to perform I/O with its platform. We propose a novel framework to determine such timing parameters from platform-independent timed models. Our framework involves two transformations. The first transformation systematically extends the platformindependent model by explicitly modeling input/output processing (e.g., sampling or interrupt-based) and the code invocation (e.g., periodic or aperiodic) mechanisms. Then, we verify if the resulting platform-specific model meets the timing requirements. In the case that the resulting model does not satisfy the timing requirements, we apply the second transformation to compensate the platform delay via adjusting the timing parameters at the code level. We formulate the adjustment mechanism using integer linear programming. If such an adjustment is feasible, generating the code with the new timing parameters guarantees the implemented system to meet the timing requirements. We validate our framework with case studies running on Patient-Controlled Analgesia (PCA) infusion pump platforms.
\end{abstract}

CCS Concepts: • Computer systems organization $\rightarrow$ Embedded software; Real-time systems; $\bullet$ Software and its engineering $\rightarrow$ Formal methods; Source code generation;

Additional Key Words and Phrases: Real-time embedded systems, code generation, timed automata, medical device, platform-independent models, platform-specific models

\section{ACM Reference format:}

Baekgyu Kim, Lu Feng, Oleg Sokolsky, and Insup Lee. 2019. Determining Timing Parameters for the Code Generation from Platform-Independent Timed Models. ACM Trans. Cyber-Phys. Syst. 3, 3, Article 28 (August 2019), 32 pages.

https://doi.org/10.1145/3230711

\footnotetext{
This article is based on the dissertation work of the first author at the University of Pennsylvania.

This work was supported in part by NSF CNS-1505799 and the Intel-NSF Partnership for Cyber-Physical Systems Security and Privacy, Global Research Laboratory Program (2013K1A1A2A02078326) through NRF, and the DGIST Research and Development Program (CPS Global Center) funded by the Ministry of Science, ICT \& Future Planning, James S. McDonnell Foundation Postdoctoral Fellowship, and Unrestricted Research Fund from Toyota InfoTechnology Center, USA.

Authors' addresses: B. Kim, 465 Bernardo Ave. Mountain View, CA 94043 United States; email: baekgyu.kim@toyota.com; L. Feng, 85 Engineer's Way, Charlottesville, VA 22904 United States; email: lu.feng@virginia.edu; O. Sokolsky and I. Lee, Department of Computer and Information Science, University of Pennsylvania, 3330 Walnut Street, Philadelphia, PA 19104; emails: \{sokolsky, lee\}@cis.upenn.edu.

Permission to make digital or hard copies of all or part of this work for personal or classroom use is granted without fee provided that copies are not made or distributed for profit or commercial advantage and that copies bear this notice and the full citation on the first page. Copyrights for components of this work owned by others than ACM must be honored. Abstracting with credit is permitted. To copy otherwise, or republish, to post on servers or to redistribute to lists, requires prior specific permission and/or a fee. Request permissions from permissions@acm.org.

(C) 2019 Association for Computing Machinery.

2378-962X/2019/08-ART28 \$15.00

https://doi.org/10.1145/3230711
}

ACM Transactions on Cyber-Physical Systems, Vol. 3, No. 3, Article 28. Publication date: August 2019. 


\section{INTRODUCTION}

Safety-critical real-time embedded systems often need to meet dependability requirements such as strict input/output timing constraints. That is, when a system receives input from the environment, it should produce the output within a bounded time. For example, when a driver presses a brake, a vehicle should stop within several seconds; when a pacemaker detects abnormal heart signals, it should generate the electrical signal within several milliseconds. Increasing complexity makes it challenging to implement an embedded software that meets such timing constraints.

Timed automata (Alur and Dill 1994) and their variants (Alur et al. 1999) have been proposed to express and verify the behavioral aspects of real-time systems. A system is described using a finitestate machine that consists of locations among which transitions happen when input and output synchronizations occur and/or when timing guards are satisfied. Once a system is modeled using timed automata, the system is verified if timing constraints are satisfied using model-checking techniques.

We consider a problem to generate software from a platform-independent model expressed in the timed-automata semantics. A platform-independent model hides the source of platform delays originating from a combination of several factors such as invocation mechanisms (periodic or aperiodic invocation) or $\mathrm{I} / \mathrm{O}$ processing mechanisms (interrupt or sampling based $\mathrm{I} / \mathrm{O}$ ). The problem is that how to incorporate the platform delays into the platform-independent model to generate software such that when integrated with the platform, the implemented system conforms to the I/O timing constraints.

There have been a number of works to add platform aspects to the high-level models. Existing work on the implementability of timed automata incorporates the platform information by explicitly modeling the execution platform (Abdellatif et al. 2010; Altisen and Tripakis 2005; Triki et al. 2013) or by modifying the semantics to reflect the implementation platform semantics (e.g., Krčál et al. (2004); Krčál and Pelánek (2005); Wulf et al. (2004)). Real-time scheduling has also been combined with timed automata in Abdeddaïm et al. (2006), and a number of automata- and actororiented scheduling interfaces have also been developed (Alur and Weiss 2008; Geilen et al. 2011; Stierand et al. 2013). However, these existing techniques consider rather restrictive platform semantics, such as assuming only a small subset of possible implementations (Altisen and Tripakis 2005) or ignoring various sources of timing delays (Abdellatif et al. 2010; Triki et al. 2013; Wulf et al. 2004).

We consider a particular form of input/output timing constraints called the delay-bound inclusion. Informally, this constraint states the min/max timing bound of input/output that a system will produce since an input (output) has been received (produced) from (to) the environment. We extend our two prior works (Kim et al. 2015a, 2015b) to propose a unified code generation framework in order for the implemented system to meet the delay-bound inclusion constraint. Given a platform chosen to execute the code, this extension enables one to incorporate the platform delays to the platform-independent model through two step transformations.

Each transformation explicitly distinguishes the two types of I/O information flows: (1) external interaction occurring in between a system and the environment; (2) internal interaction occurring in between software and a platform (i.e., software and a platform comprise a system). The platformindependent model expresses the external interaction only. By doing so, the delay-bound inclusion constraint is verified without knowing which platform will be used in the end.

The first transformation incorporates the platform delays by constructing the platform-specific model, provided with a platform that is selected to implement the system. This transformation is performed by exposing the internal interaction to the platform-independent model by desynchronizing the platform-independent I/O in a systematic way. For example, the invocation mechanism 
of software, such as periodic or aperiodic invocation, and I/O processing mechanisms, such as sampling or interrupt-based $\mathrm{I} / \mathrm{O}$, are explicitly modeled to express the source of platform delays that were hidden in the platform-independent model. However, the timing guards of the platformindependent model remains unchanged, so this transformation typically prolongs the I/O delay due to the additional platform delays. Therefore, the delay-bound inclusion constraint may or may not be satisfied depending on the amount of platform delays affecting the original behavior of the platform-independent model. As long as the constraint verifies, it is ok to generate the code, otherwise the second transformation is necessary.

In case the constraint does not verify, the second transformation compensates the platform delays from the platform-independent model. The major cause of the constraint violation is because the platform delay is not small enough to guarantee the delay-bound inclusion constraint. The second transformation aims at finding if there is some room to compensate the platform delays by changing the timing guards of the platform-independent model. This transformation is performed by formulating the compensation procedure using integer linear programming (ILP). If there exists a feasible solution (i.e., new timing parameters of the platform-independent model), then the code can be generated using the new timing parameters. When the code is integrated with the platform, the implementation guarantees to conform to the delay-bound inclusion constraint.

Our contributions are as follows:

- A categorization and formalization of the interaction among a code, a platform, and an environment based on Parnas' four variable formalism (Parnas and Madey 1995).

- A model transformation that systematically exposes platform-specific I/O interaction details to the platform-independent model.

-A model transformation that automatically adjusts timing parameters for the generated code to meet the delay-bound inclusion constraint.

- An infusion pump case study that demonstrates that the generated code conforms to the delay-bound constraint.

- An experiment result that shows how much complex platform-independent model can be handled in the proposed framework in terms of computation time required to adjust the timing parameters.

This section is organized as follows. We give the problem statement and approach overview in Section 2 and Section 3. In Section 4 and Section 5, we give the details of the two model transformations. We present the case study and experiment result in Section 6. The related work is presented in Section 7 and the conclusion is given in Section 8.

\section{THE PROBLEM STATEMENT}

\subsection{Motivating Example}

An infusion pump is a safety-critical medical device that injects drugs into a patient's body in a controlled manner for medical purposes, such as diabetes treatments or anesthesia. Its hardware platform is equipped with sensors (e.g., a bolus request button) and actuators (e.g., a pump motor) to interact with its physical environment (e.g., a patient). For example, a patient presses a bolus request button to request an additional amount of drugs; a pump rotates a pump-motor that generates physical forces to move a loaded syringe so that drug can flow from the syringe to the patient. The software operating on the platform reads sensor inputs (e.g., a patient's bolus requests) and writes actuator outputs (e.g., the rotation of a pump motor) to make infusion administration processes happen. 


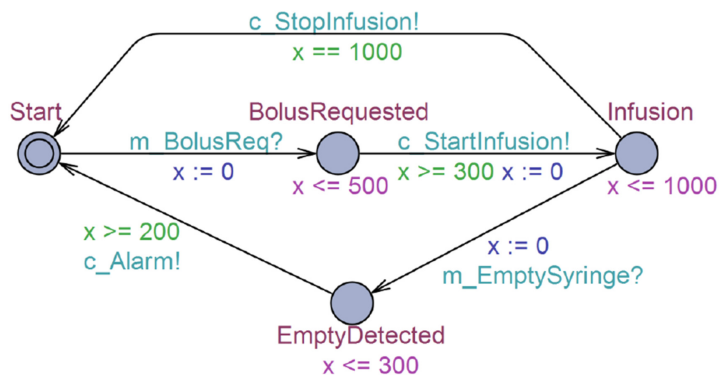

(1) $\mathrm{M}$

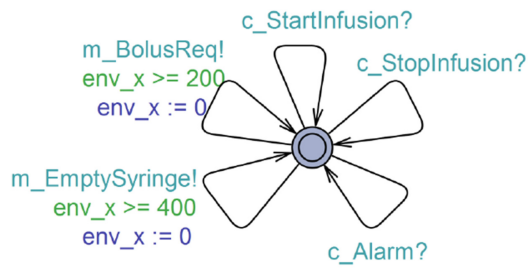

(2)ENV

Fig. 1. The example of the platform-independent model for the infusion pump system.

Consider the following timing requirement from Food and Administration (2009):

-(REQ1) "When a patient requests a bolus, a bolus infusion should start within 500ms."

Figure 1 shows an UPPAAL model that abstracts the timed behavior of the infusion pump system and its environment. We follow the definition and the operational semantics defined in G. Behrmann and Larsen (2004), and give informal semantics that are relevant to the rest of the article. $M$ consists of four locations that abstract the operational modes of an infusion pump. Start is a mode where the pump waits for a bolus request from a patient; BolusRequested is a mode where the pump calculates a right amount of a bolus before delivering it to the patient; Infusion is a mode where the pump is delivering a drug to the patient; EmptyDetected is a mode where the pump detects the empty syringe. $M$ models the system using a clock variable $(x)$ that continuously increases over time. A clock variable can be associated with invariants, guard conditions, or clock resets to express appropriate timing semantics of the model, and here are the examples: $x \leq 500$ is associated with the location BolusRequested as an invariant, which means a system can stay in the location as long as the clock variable is less than or equal to 500 time units. $x \geq 300$ is associated with the transition from BolusRequested to Infusion, which means that a system can take the transition when the clock variable $x$ is equal to or greater than 300 time units. On this transition, $x:=0$ means that the clock is reset to zero when it enters the new location Infusion.

The model also has synchronizations between two automata, $M$ and $E N V$, using input synchronizations ( $m$-BolusReq and $m$-EmptySyringe) and output synchronizations (c-StartInfusion, $c$-StopInfusion, and c-Alarm). A synchronization is associated with a transition to express reaction to an input or output event occurrence. When the synchronization matches between two transitions, both automata can take the corresponding transitions simultaneously.

\subsection{Relationship Among the Platform-Independent Model, a Code, and a Platform}

Figure 2 shows an overview of relations between a platform-independent model, a code, and a platform. We adopt Parnas' four-variable model (Parnas and Madey 1995) to define the boundaries of an implemented system (shown in the right side of the figure). In the four-variable model, monitored $\left(m_{a}\right)$ and controlled $\left(c_{b}\right)$ variables characterize changes of physical environmental quantities; on the other hand, input $\left(i_{a}\right)$ and output $\left(o_{b}\right)$ variables characterize software behavior that interact with the physical environment through input/output devices (i.e., platforms in our work). Based on this variable mapping, we define the boundaries of an implemented system to formalize the problem: io-boundary that separates a platform and the code, and $m c$-boundary that separates an environment and a platform.

\footnotetext{
${ }^{1}$ Note that the specific timing parameter $(500 \mathrm{~ms})$ is added to the original requirement to explain our work.
} 


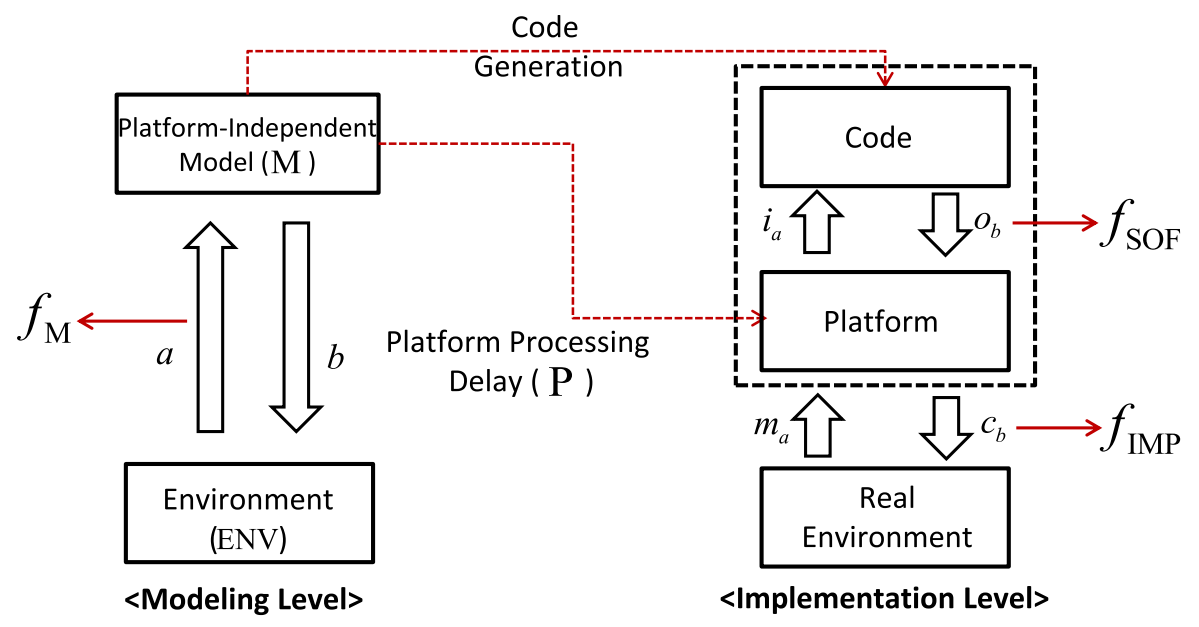

Fig. 2. Mapping from the platform-independent model to the implementation.

Note that the model in Figure 1 is platform-independent in a sense that it only expresses the external interaction occurring between the system and the environment. For example, the model expresses the timing when the output $c$-StartInfusion should be produced since the input $m$-BolusReq has been received (at the $m c$-boundary), without expressing what internal interactions (at the ioboundary) between software and a platform make such I/O information flow possible.

\subsection{Problem Statement}

We first introduce three functions, $f_{\mathrm{M}}, f_{\mathrm{SOF}}$, and $f_{\mathrm{IMP}}$, to characterize the $\mathrm{min} / \mathrm{max}$ delay-bounds of a pair of I/O events in the system model, io-boundary and $m c$-boundary, respectively. $f_{M}(a, b)$ is a function that is applied to the model level, and formally, we define $f_{\mathrm{M}}(a, b)$ as the $\min / \mathrm{max}$ delay-bounds of simple paths (i.e., those without cycles) starting from the transition $a$ and ending with the transition $b$ in the platform-independent model $M$. For example, the timing constraint REQ1 can be formally denoted by $f_{M}(m-$ BolusRequest, $c-$ StartInfusion $)=[300,500]$, representing that the output transition c-StartInfusion shall be taken in between 300 and 500 time units after taking the input transition $m$-BolusReq in Figure $1 .^{2}$ We can show that REQ1 is satisfied in this model by describing REQ1 as a logic formula, which states the maximum delay of two successive synchronizations-m-BolusReq followed by $c$-StartInfusion-does not exceed 500 time units, and verifying it using model checking. Given a simple path $p$, we can also write $f_{\mathrm{M}}(p)$.

On the other hand, $f_{\mathrm{SOF}}(a, b)$ and $f_{\mathrm{IMP}}(a, b)$ are the functions applied to the implementation level. An event defined in $M$ is mapped to the two separate events at the implementation level: one occurring at the $m c$-boundary and another occurring at the io-boundary. $f_{\mathrm{IMP}}(a, b)$ is the $\mathrm{min} / \mathrm{max}$ delay-bound of a pair of events $m_{a}$ and $c_{a}$ that occur at the $m c$-boundary. In case the event $a$ or $b$ of $M$ is an input, we assume that there will be a corresponding input event, $i_{a}$ or $i_{b}$, that needs to be read by a code at the io-boundary. Similarly, in case the event $a$ or $b$ of $M$ is an output, we assume that there was a corresponding output, $o_{a}$ or $o_{b}$, that has been written by a code at the io-boundary. $f_{\mathrm{SOF}}(a, b)$ is the $\mathrm{min} / \mathrm{max}$ delay bound of such a pair of events that occur at the io-boundary. To this end, we define the delay-bound inclusion constraint as follows:

\footnotetext{
${ }^{2}$ Note that this example only includes a single simple path between the two transitions. In general, there may be multiple simple paths between two transitions. In this case, the timing requirements should be satisfied for all such simple paths.
} 


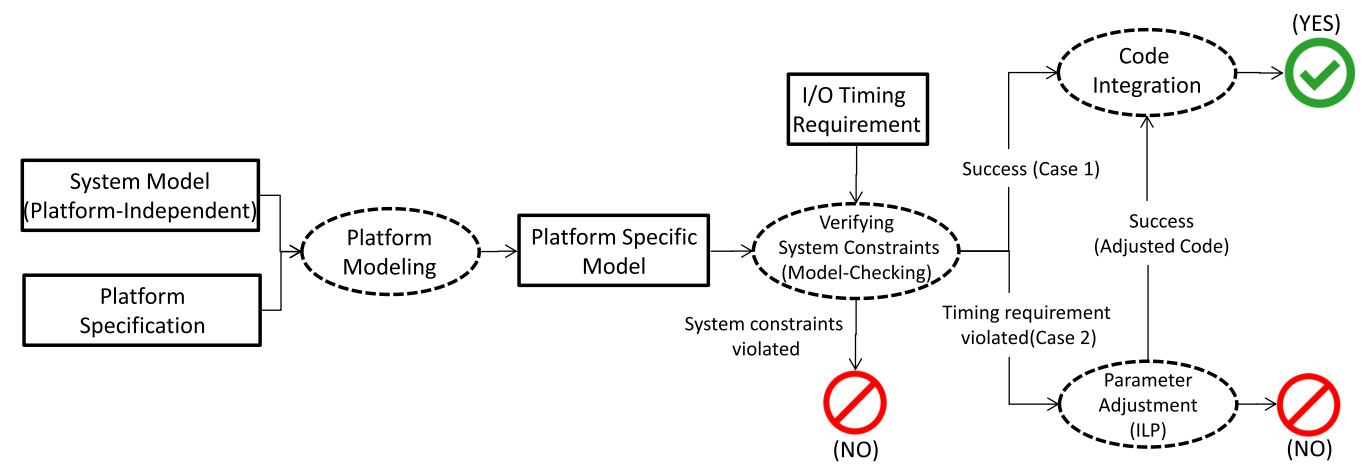

Fig. 3. The proposed verification and code generation framework.

Definition 2.1 (Delay-Bound Inclusion Constraint). A system implementation preserves the delaybound inclusion constraint with respect to the corresponding system model iff:

- the minimum delay bound of $f_{\mathrm{IMP}}$ for any pair of $\mathrm{I} / \mathrm{O}$ events at the $m c$-boundary is no less than that of $f_{M}$;

- the maximum delay bound of $f_{\text {IMP }}$ for any pair of I/O events at the $m c$-boundary is no greater than that of $f_{M}$.

Formally, we denote the constraint satisfaction by $f_{\mathrm{IMP}}(a, b) \subset f_{\mathrm{M}}(a, b)$ iff $f_{\mathrm{IMP}}^{\min }(a, b) \geq f_{\mathrm{M}}^{\min }(a, b)$ and $f_{\mathrm{IMP}}^{\max }(a, b) \leq f_{\mathrm{M}}^{\max }(a, b)$ for any pair of $\mathrm{I} / \mathrm{O}$ transitions (events) $a$ and $b$.

The problem is that given a platform that incurs additional I/O processing delays, how to generate a code in a way that satisfies the delay-bound inclusion constraint.

\section{APPROACH OVERVIEW}

Our goal is to systematically expose the internal interactions occurring at the io-boundary to the platform-independent model, and then to determine the timing parameters to generate the code for a given platform in a way that meets the delay-bound inclusion constraint. Figure 3 illustrates the proposed code generation framework. We assume that a platform-independent model that is not tied to a particular platform is given. Suppose a platform is selected that will execute a code. In order to generate the code from the platform-independent model, one needs to determine if the timing parameters of the platform-independent model (e.g., invariant or guard conditions such as 300 and 500 in Figure 1) can be used as they are or should be changed to take into account the platform delays.

We previously proposed two independent approaches. The first approach was to systematically extend the platform-independent model into the platform-specific model that is to be verified against the timing requirements (Kim et al. 2015a). This framework checks if the original parameters of the platform-independent models can be used to generate the code, but does not change the parameters in case the platform-specific model does not verify the timing requirements. The second approach was to systematically adjust such timing parameters of the platform-independent model so that the generated code can be integrated with the platform in a way that meets the timing requirements (Kim et al. 2015b). In comparison to those prior works, the main contribution of this article is to propose a unified framework that allows one to apply those model transformations under different conditions to determine the timing parameters for the code generation purposes.

The following two transformations intend to determine such timing parameters under the unified framework. The first transformation utilizes the information of the platform specification to 
explicitly model the way in which the platform supports the execution of the code to interact with the environment. This information includes the invocation mechanism, such as periodic or aperiodic invocation, and the I/O processing mechanisms, such as interrupt-based or samplingbased mechanism. Given such platform information, this transformation performs the desynchronization of I/O transitions of the platform-independent model. Each I/O synchronization of the platform-independent model is decomposed into the two types of I/O transitions; one type is the transition corresponding to $m c$-boundary $\mathrm{I} / \mathrm{O}$ and another type is the transition corresponding to io-boundary I/O. Such desynchronization makes a gap between the platform-independent model and environment. This gap is filled by modeling the platform explicitly in terms of both transitions to complete the information flow.

In the platform-specific model, the delay may be unbounded. For example, the platform is not fast enough to process the $m c$-boundary I/O events, so some events may not have a chance to reach to the io-boundary. We introduce the system constraints that should be satisfied to ensure the boundedness of the I/O delay. The model checking verifies if the system holds the system constraints or not. If the system constraints are verified, then the $\mathrm{I} / \mathrm{O}$ delay is bounded and its bound can be calculated. However, the boundedness of I/O delay does not necessarily imply the conformance of the timing requirements. If such a platform delay is added in a way the resulting I/O delay of the platform-specific model stays within an acceptable range, the timing requirement is not violated; otherwise, the timing requirement is violated, and we proceed to the second transformation.

The second transformation aims at adjusting the timing parameters of the platform-independent model. Since the first transformation does not require one to change the timing parameters of the platform-independent model, it only adds the platform delays so the delay-inclusion constraint may or may not hold. However, in case the system does not conform to the timing requirements, we may still have a chance to generate the code by adjusting the timing parameters of the platformindependent model. We formulate this adjustment mechanism in terms of ILP and show how to find new timing parameters. If one can find new timing parameters successfully, the code can be generated and integrated with the platform in a way that the implementation meets the delaybound inclusion constraint.

\section{MODEL TRANSFORMATION: PART 1}

The platform-independent model in Figure 1 expresses the information flow in terms of the input and output occurring at the $m c$-boundary only. For example, when a system receives a bolus request from a patient ( $m$-BolusReq synchronization), the system should start the infusion (c-StartInfusion synchronization) in between 300 and 500 time units. This implies that the two processing steps should be completed no earlier than 300 and no later than 500 time units as illustrated in Figure 2: A platform processing includes reading the environmental input $(m)$ and converting it in the form of the code input $(i)$; reading the code output $(o)$ and converting it in the form of the environmental output $(c)$. A code processing includes reading the code input $(i)$ and computes the appropriate output $(o)$ and writing it to the platform. To generate a code that meets the delay-bound inclusion constraint, it is necessary to determine the timing when an input $(i)$ has to be read from a platform and when an output $(o)$ has to be written to the platform by taking into account the platform delay. We define such platform delay as follows.

Definition 4.1 (Platform Delay). For any I/O transition $a_{k}$ of the platform-independent model, the platform delay $\mathrm{P}\left(a_{k}\right)=\left[\delta_{k}^{\min }, \delta_{k}^{\max }\right]$, where $\delta_{k}^{\min }$ and $\delta_{k}^{\max }$ are the minimum and maximum times needed for the platform to process the event $a_{k}$, respectively.

In the later sections, we introduce several factors that affect the platform delay (Section 4.1) and how the upper-bound of the platform delay can be calculated (Section 4.3) or measured through 
Table 1. The Categorization of the Platform-to-Environment Interaction $(m, c)$

\begin{tabular}{|c|c|c|c|}
\hline $\begin{array}{l}\text { Interaction } \\
\text { Type }\end{array}$ & $\begin{array}{l}\text { Signal } \\
\text { Type }\end{array}$ & $\begin{array}{l}\text { Read } \\
\text { Policy }\end{array}$ & $\begin{array}{l}\text { Platform Specific Parameters } \\
\qquad(P m c)\end{array}$ \\
\hline \multirow{6}{*}{$\begin{array}{l}\text { Input Interaction } \\
\text { (Env->Platform) }\end{array}$} & $\begin{array}{l}\text { Pulse Signal } \\
\text { (Type 1) }\end{array}$ & $\begin{array}{c}\text { Interrupt } \\
\text { (Rising Edge) }\end{array}$ & $\begin{array}{l}\text { (1) Min/Max input processing delay } \\
\text { (2) Input minimum inter-arrival time (Env) }\end{array}$ \\
\hline & \multirow{3}{*}{$\begin{array}{l}\text { Sustained Signal } \\
\text { (Type 2) }\end{array}$} & $\begin{array}{c}\text { Interrupt } \\
\text { (Rising Edge) }\end{array}$ & \multirow{2}{*}{$\begin{array}{l}\text { (1) Min/Max input processing delay } \\
\text { (2) Input minimum inter-arrival time (Env) } \\
\text { (3) Input sustained duration (Env) }\end{array}$} \\
\hline & & $\begin{array}{c}\text { Interrupt } \\
\text { (Falling Edge) }\end{array}$ & \\
\hline & & Polling & $\begin{array}{l}\text { (1) Min/Max input processing delay } \\
\text { (2) Input sustained duration (Env) } \\
\text { (3) Input minimum inter-arrival time (Env) } \\
\text { (4) Polling Interval }\end{array}$ \\
\hline & \multirow{2}{*}{$\begin{array}{l}\text { Sustained Signal } \\
\text { Until Read (Type 3) }\end{array}$} & $\begin{array}{c}\text { Interrupt } \\
\text { (Rising Edge) }\end{array}$ & $\begin{array}{l}\text { (1) Min/Max input processing delay } \\
\text { (2) Input minimum inter-arrival time (Env) }\end{array}$ \\
\hline & & Polling & $\begin{array}{l}\text { (1) Min/Max input processing delay } \\
\text { (2) Polling Interval } \\
\text { (3) Input minimum inter-arrival time (Env) }\end{array}$ \\
\hline \multirow{5}{*}{$\begin{array}{c}\text { Output } \\
\text { Interaction } \\
\text { (Platform->Env) }\end{array}$} & Pulse Signal (Type 1) & - & \multirow{5}{*}{ (1) Min/Max output processing delay } \\
\hline & & Non-Blocking & \\
\hline & Sustanted signal ( Iype 2) & Blocking & \\
\hline & \multirow{2}{*}{$\begin{array}{l}\text { Sustained Signal } \\
\text { Until Read (Type 3) }\end{array}$} & Non-Blocking & \\
\hline & & Blocking & \\
\hline
\end{tabular}

testing (Section 6). In addition, we explain how to check the delay-bound inclusion property if the code is generated using the original timing parameters of the platform-independent model.

\subsection{The Categorization of the mc-Boundary and io-Boundary Interactions}

When a platform-independent model is implemented on a given platform, several aspects at the $m c$-boundary contribute to the platform delay. Hence, it is necessary to understand those platformspecific aspects to generate code in a way that meets the delay-bound inclusion constraint. In this subsection, we categorize those aspects in terms of $m c$-boundary and io-boundary interactions.

We characterize the external interactions between the platform and the environment in terms of the types of physical signals and the way in which a platform reads or writes them. Table 1 categorizes the interactions according to this criteria.

The input interaction subcategory includes how a platform reads signals (or values) that are changed by the environment. It is categorized based on the types of signals generated from the environment. There are three types of input signals generated by the environment illustrated in Figure 4(a): pulse signals (Type 1), signals with a sustained duration (Type 2), and signals sustained until being read (Type 3$).{ }^{3}$ The platform can read the signals using either a polling or an interruptbased mechanism.

A pulse signal does not have a sustained duration, i.e., its sustained duration is too short to be captured through a polling-based mechanism. Therefore, a platform can only read the signal using an interrupt-based mechanism, in which an interrupt service routine is automatically called for processing the input signal whenever a signal change is detected on a sensor. For instance, an

\footnotetext{
${ }^{3}$ We mainly considered a discrete signal in our categorization of the signal types. Considering continuous signal types will give more complex categorization and it is out of the scope of this article.
} 
(a) Three types of $m$-variables

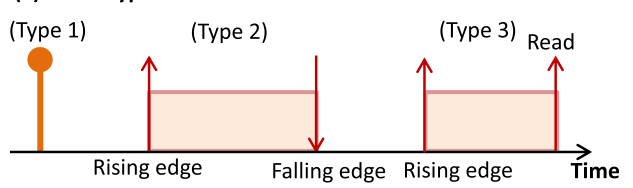

(b) Inter-arrival time of $\mathbf{m}$-variables

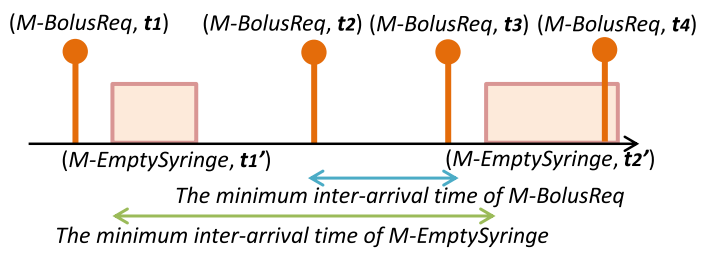

Fig. 4. The environmental signal types.

infusion pump needs to detect drug-drops using a drop sensor to precisely calculate the volume of drugs infused; since a drug-drop passes the sensor very fast, this detection is typically done using an interrupt-based mechanism. A signal with a sustained duration has a non-zero sustained duration; once the signal is triggered, it is sustained for a certain amount of time duration, and disappears. Therefore, a platform has options to read the signal, either an interrupt-based or a polling-based scheme. In the interrupt-based scheme, a platform has two additional options in choosing the timing of signal reading, a rising-edge or a falling-edge. For example, an infusion pump should detect a pressed state of a bolus request button or a closed state of an empty syringe sensor; granularity of the sustained time for both signals is far greater than a drug-drop so that they can be detected through a polling-based scheme. A signal sustained until read is sustained until the signal is read by a platform. Like Type- 2 signal, a platform can read the signal through either an interrupt-based scheme or a polling-based scheme. However, in the case of the interrupt-based scheme, a platform is only able to read the signal at its rising-edge since a falling-edge is triggered immediately when a platform reads it. For example, the environment is another system that keeps an item in a buffer until a platform reads the item. The output interaction subcategory includes implementation schemes that a platform writes signals to the environment, which is typically performed through actuators. Like the input interaction, the output interaction considers three different types of environmental signals. Unlike the input interaction, however, a platform can be blocked or non-blocked over the outputs that are currently being produced. In the former case, a platform cannot produce another output until the current output is read by the environment or disappears due to time-out. In the latter case, a platform can produce another output at any time, regardless of the output currently produced.

io-Boundary Interactions. We characterize the internal interactions in terms of the way in which the platform invokes the code and how the platform exchanges events (or data) with the code. Table 2 categorizes the interactions according to this criteria.

In order for a code to execute its computation, it needs to be invoked by some mechanisms implemented by the platform. The invocation type can be either periodic or aperiodic that enables a code to be invoked every pre-defined period or by some events generated from the platform. For example, in some real-time operating systems, a code calls API with the period parameter to let the operating system know the specific timing to invoke a code. A code can be linked with a blocking message queue that lets the code start execution whenever a message arrives.

Upon invocation either periodically or aperiodically, a code needs to read input or to write output from/to the platform. Such communication can be done through either shared variables or queues. In the case of using a shared variable, an event is overwritten to the previous event, but in the case of using a queue, all events are kept separately as long as the queue is non-full. Since a queue may contain more than one event, a code has the options as to how many events need to be read per invocation. 
Table 2. The Categorization of the Platform-to-Code Interaction $(i, o)$

\begin{tabular}{|c|c|c|c|c|}
\hline $\begin{array}{l}\text { Invocation } \\
\text { Type }\end{array}$ & $\begin{array}{l}\text { Interaction } \\
\text { Type }\end{array}$ & $\begin{array}{c}\text { Communication } \\
\text { Mechanism }\end{array}$ & $\begin{array}{l}\text { Read } \\
\text { Policy }\end{array}$ & $\begin{array}{l}\text { Platform-Specific } \\
\text { Parameters (Pio) }\end{array}$ \\
\hline \multirow{6}{*}{ Periodic } & \multirow{3}{*}{$\begin{array}{c}\text { Input } \\
\text { Interaction } \\
\text { (Platform-> } \\
\text { Code) }\end{array}$} & Shared variable & - & (1) Invocation period \\
\hline & & \multirow{2}{*}{ Queues } & $\begin{array}{l}\text { Read One } \\
\text { (Code) }\end{array}$ & \multirow{2}{*}{$\begin{array}{l}\text { (1) Invocation period } \\
\text { (2) Input queue size }\end{array}$} \\
\hline & & & $\begin{array}{l}\text { Read All } \\
\text { (Code) }\end{array}$ & \\
\hline & \multirow{3}{*}{$\begin{array}{c}\text { Output } \\
\text { Interaction } \\
\text { (Code -> } \\
\text { Platform) }\end{array}$} & Shared variable & - & $\begin{array}{l}\text { (1) Invocation period } \\
\text { (2) Polling interval }\end{array}$ \\
\hline & & \multirow{2}{*}{ Queues } & $\begin{array}{l}\text { Read One } \\
\text { (Platform) }\end{array}$ & \multirow{2}{*}{$\begin{array}{l}\text { (1) Invocation period } \\
\text { (2) Output queue size } \\
\text { (3) Polling interval }\end{array}$} \\
\hline & & & $\begin{array}{l}\text { Read All } \\
\text { (Platform) }\end{array}$ & \\
\hline \multirow{6}{*}{ Aperiodic } & \multirow{3}{*}{$\begin{array}{c}\text { Input } \\
\text { Interaction } \\
\text { (Platform } \rightarrow \\
\text { Code) }\end{array}$} & Shared variable & - & (1) Aperiodic trigger event \\
\hline & & \multirow{2}{*}{ Queues } & $\begin{array}{l}\text { Read One } \\
\text { (Code) }\end{array}$ & \multirow{2}{*}{$\begin{array}{l}\text { (1) Aperiodic trigger event } \\
\text { (2) Input queue size }\end{array}$} \\
\hline & & & $\begin{array}{l}\text { Read All } \\
\text { (Code) }\end{array}$ & \\
\hline & \multirow{3}{*}{$\begin{array}{c}\text { Output } \\
\text { Interaction } \\
\text { (Code -> } \\
\text { Platform) }\end{array}$} & Shared variable & - & $\begin{array}{l}\text { (1) Aperiodic trigger event } \\
\text { (2) Polling interval }\end{array}$ \\
\hline & & \multirow{2}{*}{ Queues } & $\begin{array}{l}\text { Read One } \\
\text { (Platform) }\end{array}$ & \multirow{2}{*}{$\begin{array}{l}\text { (1) Aperiodic trigger event } \\
\text { (2) Output queue size } \\
\text { (3) Polling interval }\end{array}$} \\
\hline & & & $\begin{array}{l}\text { Read All } \\
\text { (Platform) }\end{array}$ & \\
\hline
\end{tabular}

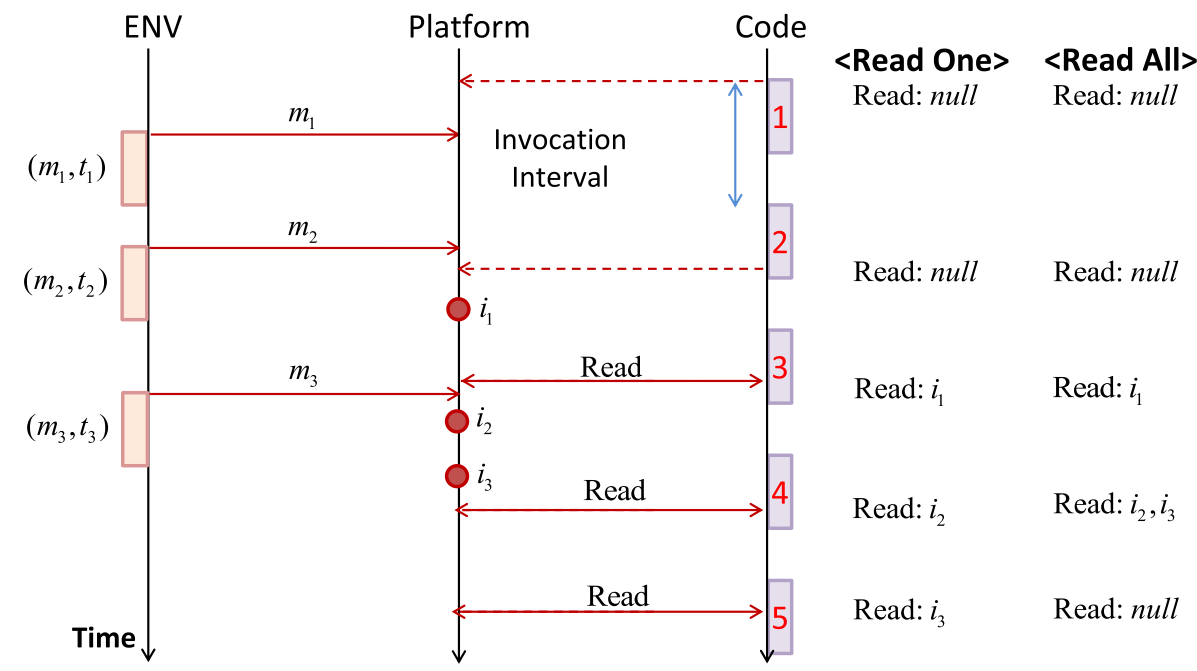

Fig. 5. The illustration of the $m c$ - and io-boundary interactions.

Figure 5 illustrates one possible io-boundary interaction. The environment generates three (Type 2) signals $\left(m_{1}, m_{2}, m_{3}\right)$ in order. The platform reads the signals using an interrupt-based mechanism on their rising edges at the $m c$-boundary and processes them; the processed input $\left(i_{1}, i_{2}, i_{3}\right)$ become available sometime after (the available timing is expressed as a red circle). Even though the inputs are available from the platform, the code cannot read them immediately due to the mismatch between the available timing of the input and the invocation timing of the code. If the input becomes available during a certain invocation period, the code may read it within the 


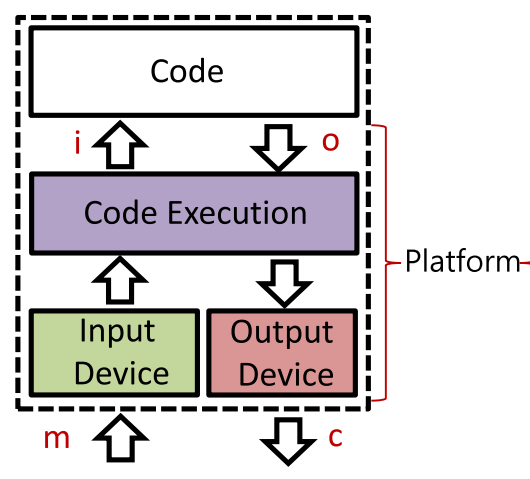

Real Environment

(a) Implementation

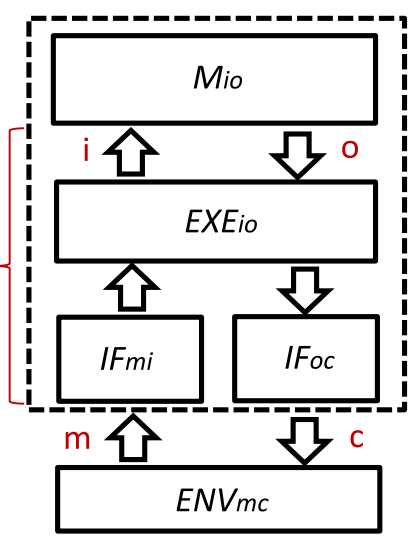

(b) Platform-Specific Model

(PSM)

Fig. 6. The mapping between (a) the implemented system and (b) its platform-specific model after the first transformation.

same invocation period (e.g., in Figure $5, i_{3}$ is available at the fourth invocation period and the code may read it at the fourth invocation period). However, the timing to read the input can be up to the immediately following invocation period (read all policicies) or the later invocation periods depending on how many inputs are in the queue (read one policy).

\subsection{The Transformation Mechanism}

The illustration in the previous subsection implies that there are several aspects that comprise the platform delay in a complex way. We introduce a model transformation to systematically expose such io-boundary interaction from the platform-independent model so that, when the code is generated from the timing parameters of the platform-independent model, one can check if the implementation meets the delay-bound constraints. In this transformation, we make two assumptions: first, the environment signal and the way in which the platform reads it are described according to the categorization in Table 1; second, the characteristic of a platform is described according to the categorization in Table 2 . The basic idea of our transformation is to desynchronize the $m c$-boundary interaction of the platform-independent model, and then to insert additional timed automata that express the invocation and the communication mechanism.

The Building Blocks of the Platform-Specific Mmodel. Figure 6 illustrates how such desynchronization happens at the platform-specific model with respect to the implemented system. The implemented system (a) interacts with its environment through the input and output devices. The input (output) device reads (writes) the environmental input (output) at the $m c$-boundary. The implemented system invokes the code using some invocation mechanisms expressed as the code execution block. Upon invocation, the code can read (write) input (output) from/to the platform.

The platform-specific model (b) characterizes such platform-specific information by inserting several building blocks. $I F_{m i}$ and $I F_{o c}$ corresponds to the input/output devices, which models the platform processing required to read or write the environmental input/output. $E X E_{i o}$ corresponds to the code execution, which models the invocation mechanism of the code. $I F_{m i}, I F_{o c}$, and $E X E_{i o}$ interact with each other to model the communication mechanism.

The Syntactic Construction of the Platform-Specific Model. The platform-independent model uses the synchronization to characterize the $m c$-boundary interaction without specifying 
(a) ENVM_BolusReq (Type 1 signal)

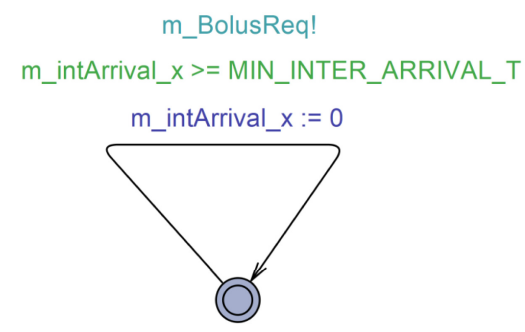

(b) ENVM_BolusReq (Type 2 signal)

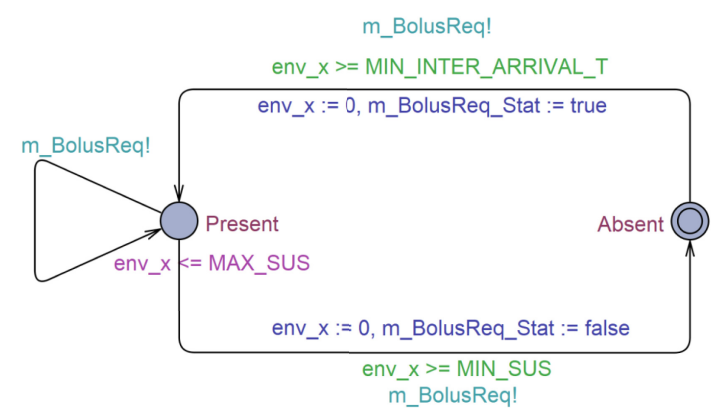

Fig. 7. The environmental model of Type 1 and Type 2 signals.

(a) IFMI_BolusReq (Interrupt-Based)

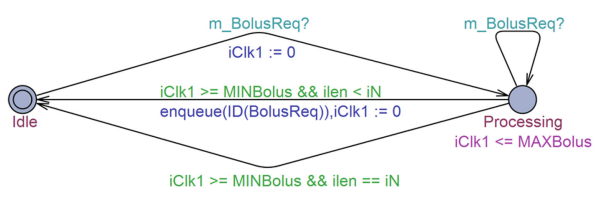

(b) IFMI_BolusReq (Polling-Based)

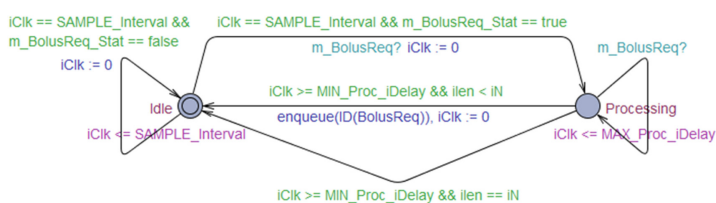

Fig. 8. The input interface automata for PSM.

the internal behavior of the code execution and input/output devices. To insert the platformspecific information, the original synchronization is renamed as the two different synchronizations; one synchronization expresses the $m c$-boundary interaction and the other synchronization expresses io-boundary interaction. For example, the original synchronization $m$-BolusReq of $M$ in Figure 1 is renamed as $i$-BolusReq to express io-boundary interaction. As a result, $M_{i o}$ and $E N V$ automata, which previously comprised the platform-independent model, become desynchronized since the two automata do not share a common synchronization due to such renaming. Then, we construct $E X E_{i o}, I F_{m i}$, and $I F_{o c}$ that synchronize with $M_{i o}$ and $E N V$ forming the complete information flow among the environment, the platform, and the code.

Figure 7 is the examples of $E N V_{m-B o l u s R e q}$ that model two types of environment signals for $m$-BolusReq (see the informal semantics of the signals in Table 1 and Figure 4). Type 1 signal is a pulse signal that does not have a sustained duration. To model this, a single location and a selftransition are constructed (a). The self-transition is associated with a guard condition to enable the transition only after a certain amount of time elapse since the transition has taken previously; this is to model the semantics of the minimum inter-arrival time between successive signals. When the transition is taken, it is synchronized with the input interface automata $\left(I F_{m i}\right.$ in Figure 8$)$ over the channel $m$-BolusReq.

Type 2 signal is a sustained signal that has non-zero sustained duration. To model this, two locations (Absent and Present) are constructed to express if the signal is absent and present (b). The transition from Absent to Present is enabled when the guard condition is satisfied to enforce the semantics of the minimum inter-arrival time. Upon taking this transition, it may be synchronized with the input interface automata over the channel $m$-BolusReq to model the interrupt policy (rising edge) or it may update the state variable $m$-BolusReq-Stat to inform the input interface automata that the signal is present and ready to read, which is used to model the polling policy. The time duration staying in Present location is limited by the associated invariant implying that the 


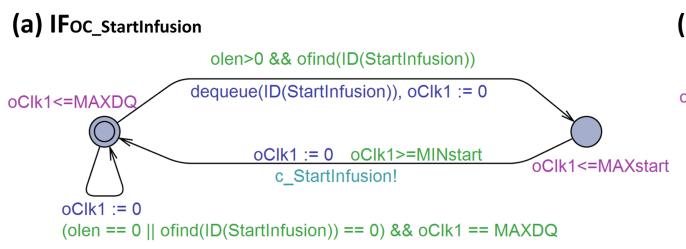

(b) IFoc_StopInfusion

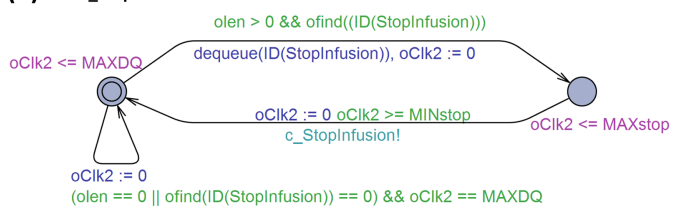

(c) IFoc_Alarm

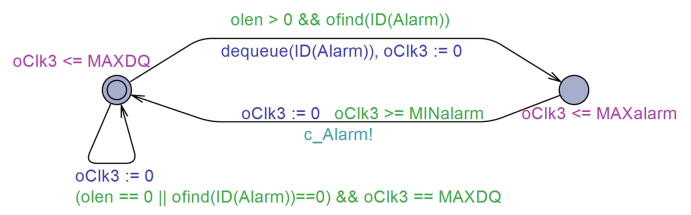

Fig. 9. The output interface automata for PSM.

signal cannot be sustained more than the maximum sustained duration. The transition from Present to Absent is taken anytime in between the minimum and the maximum sustained duration. ${ }^{4}$

Figure 8 is the example input interface automata $\left(I F_{m i}\right)$ that are constructed for $m$-BolusReq and synchronized with the environment model in Figure 7. Two read policies, interrupt and polling, are modeled independently according to Table 1; each model allows the platform to read the environment input according to the respective policy. Both policies are modeled with two locations to express the platform status when no input processing is in progress (Idle) and input processing is in progress (Processing). The key difference between the two lies on whether the timing when the platform can read the environment signal is determined by the environment or by the platform itself. In the case of the interrupt policy, the transition from Idle to Processing is enabled without any guard condition imposed by the input interface. This implies that the timing of the synchronization is determined by the environment, but not by the platform.

In the case of the polling policy, the transition from Idle to Processing has an additional guard condition imposing the synchronization may occur only if a new sampling period starts ${ }^{5}$; when the input is available from the environment at that time, the platform may read the input. This implies that, the timing of the synchronization is determined not only by the environment, but also by the platform. The rest of the model is similar for both policies. Once reading the environment input, $I F_{m i}$ converts it into the program input, which consumes some processing time, and then puts it in the queue. The output interface is also constructed similarly in Figure 9 but to realize the opposite information flow from the platform to the environment.

Figure 10 is the example $E X E_{i o}$ automata that expresses the invocation mechanism of the code. We consider the periodic invocation and queues as a communication mechanism from Table 2 . The code, initially waiting for the invocation, becomes active when the invocation period starts. Then, the code reads the input, computes the output, and writes the output to the queue as follows. If there is any input in the queue, it performs the corresponding synchronization with $M_{i o}$, which indicates the code has read the input. After sometime later, it performs the output synchronization in a similar fashion to express the opposite information flow. Such reading, computing, and writing steps should be completed within the worst-case execution time that is assumed to be known $a$ priori. After completing those steps, the code becomes idle waiting for another invocation. The

\footnotetext{
${ }^{4}$ Figure 7(b) expresses three possible options in reading the sustained signal together (i.e., interrupt policy (rising and falling edges) and polling policy) to avoid redundant figures. When the model is constructed, only one option is used. For example, when the interrupt with a rising edge is used, the upper $m$-BolusReq is used while the bottom $m$-BolusReq is removed. ${ }^{5}$ Note that this condition can be easily modified in several different ways. For example, one can change the guard condition to read the signal anytime during a sampling period or the end of a sampling period and so on.
} 


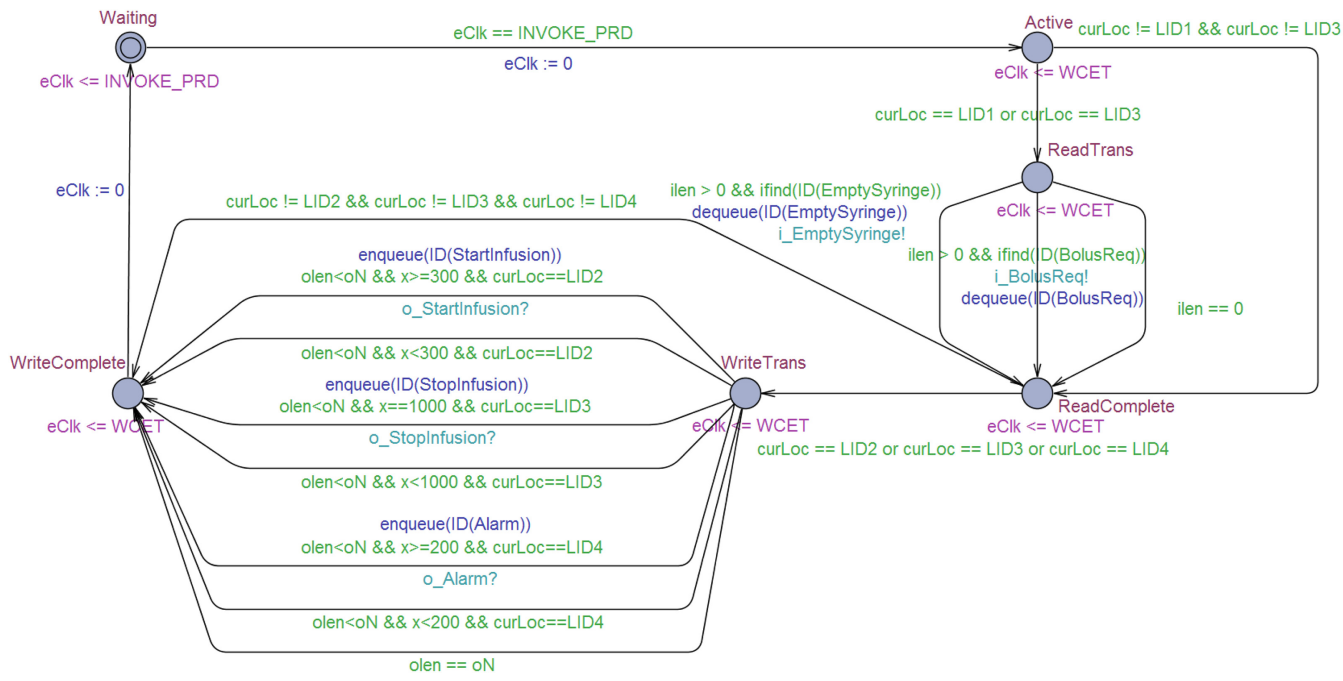

Fig. 10. The code execution model for PSM.

algorithms to perform such syntactic construction are explained in our prior work (Kim et al. 2015a).

Figure 11 illustrates the timing semantics of the platform-specific model in comparison to the platform-independent model. The platform-independent model expresses the input and output timing at the $m c$-boundary using the two synchronizations $m_{k}$ and $c_{k}$ as illustrated in (a). However, the platform-specific model exposes the internal interaction using additional synchronizations $i_{k}$ and $o_{k}$. When the environment generates the input $\left(m_{k}\right)$, it will be first read by the input device automata $\left(I F_{m i}\right) . I F_{m i}$ will then process the environment input and put it in the queue. Note that the code whose behavior is expressed as $M_{i o}$ does not read the input immediately. Sometime later, the code is invoked by the invocation mechanism $\left(E X E_{i o}\right)$; upon invocation, if there exists any input in the queue, the code will read the input $\left(i_{k}\right)$. Then, the code performs the internal computation based on the input (e.g., computing the output value) and writes it to the queue. Similarly, the output device does not read the program output immediately. Sometime later, the output device reads the value from the queue $\left(o_{k}\right)$, processes it, and writes it to the environment $\left(c_{k}\right)$.

Note that the delay bound of the code processing in $M_{i o}$ directly comes from the timing parameters of the platform-independent model. For example, in Figure 1, the platform-independent model allows the delay bound of 300 and 500 time units in between m-BolusReq and c-StartInfusion. The corresponding platform-specific model allows the same amount of delay (i.e., 300 and 500 time units) in between the corresponding internal I/O transitions (i.e., $i$-BolusReq and o-StartInfusion). Constructing the platform-specific model without changing the original timing parameters would increase the delay bound at the $m c$-boundary of the platform-specific model since the platform delay is added up to the code processing delay. We analyze the impact of the platform delay in the following subsection.

\subsection{The Property of the Platform-Specific Model}

We first distinguish the three delay types occurring in the platform-specific model as follows.

(1) M-C Delay $\Delta_{m c}$ : specifies the maximum time passage from the instant the environment triggers an input $\left(m_{j}, t_{m_{j}}\right)$ until the instant the environment observes an output $\left(c_{j}, t_{c_{j}}\right)$ 

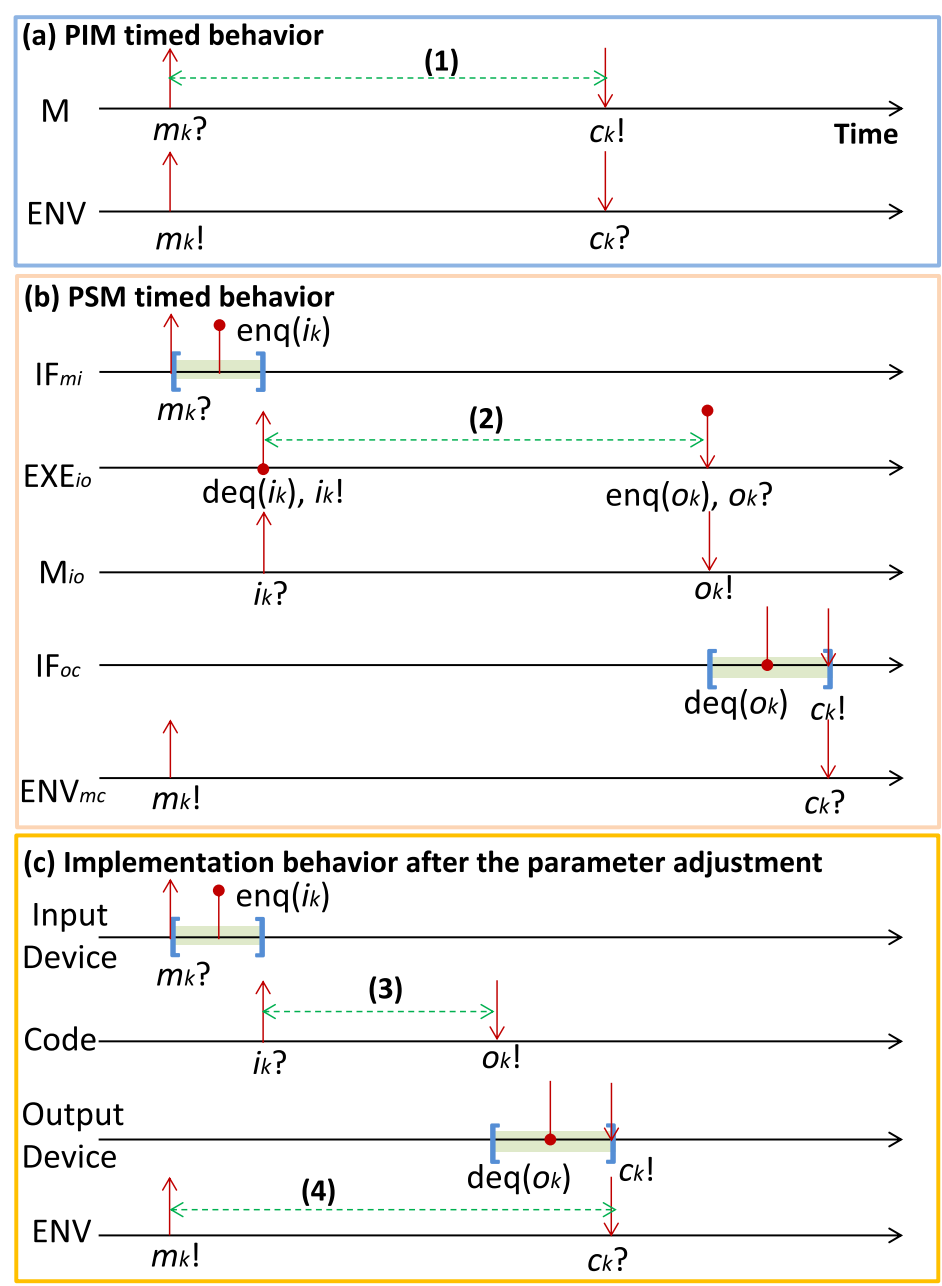

Fig. 11. The illustration of the timed behaviors of the PIM and PSM and implemented system (after the parameter adjustment).

at the $m c$-boundary, i.e., $\Delta_{m c}=t_{c_{j}}-t_{m_{j}}$; this delay is illustrated in Figure 11 as the time passage between the two synchronizations $\left(m_{k}\right.$ ! and $c_{k}$ ?) of $E N V_{m c}$.

(2) Input-Delay $\Delta_{m i}$ : specifies the maximum time passage from the instant the environment triggers an input $\left(m_{j}, t_{m_{j}}\right)$ at the $m c$-boundary until the instant the code reads the input $\left(i_{j}, t_{i_{j}}\right)$ at the io-boundary, i.e., $\Delta_{m i}=t_{i_{j}}-t_{m_{j}}$; this delay is illustrated in Figure 11 as the guarded section of $I F_{m i}$.

(3) Output-Delay $\Delta_{o c}$ : specifies the maximum time passage from the instant the code produces an output $\left(o_{j}, t_{o_{j}}\right)$ at the io-boundary until the instant the environment observes the output $\left(c_{j}, t_{c_{j}}\right)$ at the $m c$-boundary, i.e., $\Delta_{o c}=t_{c_{j}}-t_{o_{j}}$; this delay is illustrated in Figure 11 as the guarded section of $I F_{o c}$.

However, if the following constraints are satisfied, then we can find the bound of the $m c$-delay. 
(Constraint 1) Detection of all input signals: Given an input pattern generated from $E N V_{m c}$, (1) $I F_{m i}$ can detect all input signals, and (2) the maximum input processing delay of $I F_{m i}$ is shorter than the minimum inter-arrival time.

(Constraint 2) No overflow of the input queue: The invocation interval of $E X E_{i o}$ should be small enough w.r.t. the input processing speed of $I F_{m i}$ so that each input can be read by $E X E_{i o}$ before the input queue overflows.

(Constraint 3) No overflow of the output queue: Given an output pattern generated by $M_{i o}$, (1) $I F_{o c}$ has sufficient processing speed to process all outputs before the output queue overflows, and make them visible to $E N V_{m c}$, and (2) $E N V_{m c}$ can read the produced outputs by $I F_{o c}$ fast enough.

(Constraint 4) No internal transition occurrences: We call a transition occurring in $M_{i o}$ an internal transition. Since $E N V_{m c}$ generates an input, $M_{i o}$ does not take internal transitions until the input is processed by $I F_{m i}$ and read by $M_{i o}$.

We can verify the constraints from the platform-specific model using the model-checking. This can be done by writing a query in the temporal logic formula, but we do not explain the details here. If all of those constraints verify, we can compute the bound of the input and output delays as shown in Lemma 4.2 .

LEMma 4.2. If the system constraints are verified in PSM, then (1) the Input-Delay is bounded by the function of all maximum platform-specific parameters ${ }^{6}$ that are used for $E N V_{m c}, I F_{m i}$, EXE $E_{i o}$; (2) the Output-Delay is bounded by the function of all maximum platform-specific parameters that are used for $E N V_{m c}, I F_{o c}$, and $E X E_{i o}$.

Proof. Refer to our prior work (Kim et al. 2015a).

Lemma 4.2 computes the input and the output delay that are originating from the platform. Our goal is to compute the $m c$-delay of the platform-specific model to see how much delay is increased w.r.t. that of the platform-independent model. Recall that $\Delta_{m i}$ and $\Delta_{o c}$ are the upper bounds of Input-Delay and Output-Delay as $\Delta_{m i}$ and $\Delta_{o c}$. Let $\Delta_{i o \text {-internal }}$ be the maximum internal delay of the PIM for processing the input and output pair $(i, o)$. The calculation of the $m c$-delay can be performed according to Lemma 4.3.

LEMma 4.3. If the system constraints are verified in PSM, then a possible value of $\Delta_{m c}^{\prime}$ for which PSM verifies is given by $\Delta_{m c}^{\prime}=\Delta_{m i}+\Delta_{o c}+\Delta_{i o-i n t e r n a l}$.

Proof. Refer to our prior work (Kim et al. 2015a).

If the implemented system is built according to the io-boundary interaction type, the actual delay of the implemented system is bounded by the calculated $m c$-delay from Lemma 4.3. If the calculated $m c$-delay is within the range of the min/max timing constraints (illustrated in Case 1 of Figure 3), then the code can be generated using the timing parameters of the platform-independent model. Otherwise, there is no guarantee for the implemented system to conform to the timing constraints.

\section{MODEL TRANSFORMATION: PART 2}

The first transformation introduced in Section 4 does not change the timing parameters of the platform-independent model for the code generation purpose. As illustrated in Figure 11, this results in the code-level delay (2) becoming the same as the $m c$-delay (1) in the platform-independent

\footnotetext{
${ }^{6}$ Those parameters are the upper-bounds on the delays occurring in each automaton of PSM (e.g., the upper-bound on the processing time of input and output in $I F_{m i}$ and $I F_{o c}$; the invocation period and the worst execution time of the code in $\left.E X E_{i o}\right)$.
} 


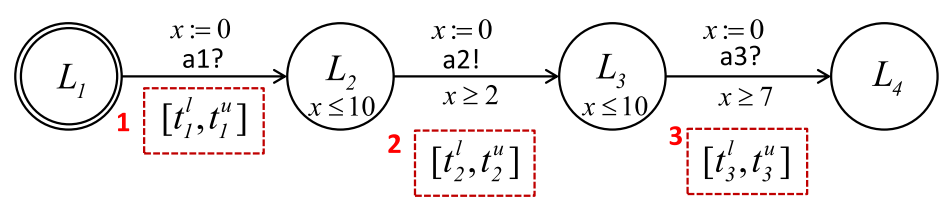

Fig. 12. The example of the variable assignment (Sequential Pattern).

model. Depending on how much the platform delay is added up, the platform-specific model may or may not preserve the delay-bound constraints at the $m c$-boundary.

In this section, we introduce the mechanism to automatically adjust the timing parameters of the platform-independent model for the code generation purpose. Intuitively, the mechanism adjusts the timing parameters in a way that the code-level delay is appropriately reduced to compensate the platform delays. For example, in Figure 11(c), the code writes the output at the io-boundary earlier than $M_{i o}$ in Figure 11(b) does. As a result, the timing of the corresponding output at the $m c-$ boundary in Figure 11(c) occurs no later than the timing of the output of the platform-independent model in Figure 11(a) (i.e., the delay-bound inclusion constraint holds). However, such an adjustment mechanism is not always possible due to several constraints; for example, no matter how much the code level delay is reduced, the delay-bound inclusion constraint cannot be satisfied if the platform delay is too big to compensate.

Our approach is to formulate this problem using ILP. ${ }^{7}$ Through this formulation, we want to know if such an adjustment is feasible or not. If feasible, the ILP solver can automatically find the new timing parameters with which the code can be generated.

\subsection{Formulation of the Linear Constraints}

We first identify the unknown variables to formulate the linear constraints. To generate the code from the platform-independent model, we need to find the unknown $\mathrm{min} / \mathrm{max}$ bound of the time interval where the code can perform the io-boundary I/O (i.e., the code reads/writes input/output from/to the platform). In the platform-independent model, each $m c$-boundary I/O (i.e., the system reads/writes input/output from/to the environment) occurs on transitions with the known $\mathrm{min} / \mathrm{max}$ timing guards. Assuming that each $\mathrm{I} / \mathrm{O}$ at the $m c$-boundary has its uniquely matched $\mathrm{I} / \mathrm{O}$ at the io-boundary, we assign two unknown variables $\left(t_{i}^{l}, t_{i}^{u}\right)$ for each I/O transition of the platform-independent model. The min/max unknown variables represent the interval the code should perform I/O at the io-boundary. Figure 12 illustrates the variable assignment. In this example platform-independent model, the four locations $\left(L_{1}, L_{2}, L_{3}, L_{4}\right)$ are connected through a sequence of $\mathrm{I} / \mathrm{O}$ transitions with the known $\mathrm{min} / \mathrm{max}$ timing guards. We associate the unknown $\min / \max$ variables $\left(t_{1}^{l}, t_{1}^{u}, t_{2}^{l}, t_{2}^{u}, t_{3}^{l}, t_{3}^{u}\right)$ for each I/O transition.

Based on this variable mapping, we consider the following types of constraints for formulation of the linear constraints.

-(Type 1) The minimum delay bound of the implemented system should be equal or greater than that of $M$ (i.e., $f_{I M P}^{\min } \geq f_{M}^{\min }$ ).

-(Type 2) The maximum delay bound of the implemented system should be equal or less than that of $M$ (i.e., $f_{1 M P}^{\max } \leq f_{M}^{\max }$ ).

-(Type 3) Each min/max delay bound of the code should be non-negative.

-(Type 4) The minimum delay-bound of the code should be equal or less than the maximum delay bound.

\footnotetext{
${ }^{7}$ Note that it is out of scope to prove whether the timing parameter adjustment is NP-complete or not. There may be more efficient algorithms to solve this problem.
} 
Type 1 and 2 constraints express the delay-bound inclusion constraint in Definition 2.1. Type 3 constraint is needed to have only positive timing parameters for the code generation (i.e., there is no case where the timing guard is negative). Type 4 constraint is needed to encode the meaning of the $\mathrm{min} / \mathrm{max}$ bound.

These constraints involve the computation of the $f_{M}$ and $f_{\text {IMP. In }}$ general, the computation of the two functions is difficult due to the complex dependency between the clock variables and I/O transition, and the computation algorithm may not terminate in the presence of the cycles (Kang and Lee 1996). However, if we restrict the timed automata to have the clock reset on every transition, $f_{\mathrm{M}}$ can be computed by applying the shortest and longest path algorithm in the graph theory (e.g., Floyd-Warshall algorithm) and detailed in our prior work (Kim et al. 2015b). All parameters to compute $f_{\mathrm{M}}$ are known a priori such as the automata structure (e.g., acyclic or cyclic automata) and the timing parameters. As a result, we can obtain two integer values of $f_{M}^{\min }$ and $f_{M}^{\max }$ for each pair of I/O. Computing $f_{I M P}$ requires one to characterize the information flow among the platform and the code. In comparison to $f_{M}, f_{I M P}$ needs the case-by-case analysis considering the event order as to how input and output occur and are processed. For example, the information flow in Figure 1 implies that the code can read the input only after the platform finishes its processing; the platform can write the output to the environment only after the code writes the output to the platform. Based on such case by case analysis, the analytic forms of $f_{\mathrm{IMP}}(i, j)$ are expressed as follows:

-Case 1: $i$ is an input event and $j$ is an output event:

$$
\left[f_{\mathrm{SOF}}^{\min }(i, j)+\left(\mathrm{P}^{\min }\left(a_{j}\right)+\mathrm{P}^{\min }\left(a_{i}\right)\right), f_{\mathrm{SOF}}^{\max }(i, j)+\left(\mathrm{P}^{\max }\left(a_{j}\right)+\mathrm{P}^{\max }\left(a_{i}\right)\right)\right] .
$$

- Case 2: $i$ is an output event and $j$ is an output event:

$$
\left[f_{\mathrm{SOF}}^{\min }(i, j)+\left(\mathrm{P}^{\min }\left(a_{j}\right)-\mathrm{P}^{\max }\left(a_{i}\right)\right), f_{\mathrm{SOF}}^{\max }(i, j)+\left(\mathrm{P}^{\max }\left(a_{j}\right)-\mathrm{P}^{\min }\left(a_{i}\right)\right)\right] .
$$

-Case 3: $i$ is an output event and $j$ is an input event:

$$
\left[f_{\mathrm{SOF}}^{\min }(i, j)-\left(\mathrm{P}^{\max }\left(a_{j}\right)+\mathrm{P}^{\max }\left(a_{i}\right)\right), f_{\mathrm{SOF}}^{\max }(i, j)-\left(\mathrm{P}^{\min }\left(a_{j}\right)+\mathrm{P}^{\min }\left(a_{i}\right)\right)\right] .
$$

-Case 4: $i$ is an input event and $j$ is an input event:

$$
\left[f_{\mathrm{SOF}}^{\min }(i, j)-\left(\mathrm{P}^{\max }\left(a_{j}\right)-\mathrm{P}^{\min }\left(a_{i}\right)\right), f_{\mathrm{SOF}}^{\max }(i, j)-\left(\mathrm{P}^{\min }\left(a_{j}\right)-\mathrm{P}^{\max }\left(a_{i}\right)\right)\right] .
$$

$f_{\mathrm{SOF}}^{\min }$ and $f_{\mathrm{SOF}}^{\max }$ are the $\min /$ max code-level delay expressed in terms of the linear combination of the unknown variables. The computation of $f_{\text {SOF }}$ is the same as the computation of $f_{M}$ except that $f_{\text {SOF }}$ involves the unknown variables. $\mathrm{P}^{\min }$ and $\mathrm{P}^{\max }$ are the $\mathrm{min} / \mathrm{max}$ platform delay that can be calculated from Lemma 4.2. Here is the example of the linear constraints for the model in Figure 12.

Example 5.1 (Linear constraints for the model in Figure 12). Figure 12 has three pairs of I/O transitions: $(1,2),(2,3),(1,3)$, and here are the linear constraints:

$$
\begin{aligned}
& -(\mathrm{C} 1 \mathrm{a}) t_{2}^{l}+\mathrm{P}^{\min }\left(a_{2}\right)+\mathrm{P}^{\min }\left(a_{1}\right) \geq 2 . \\
& -(\mathrm{C} 1 \mathrm{~b}) t_{2}^{u}+\mathrm{P}^{\max }\left(a_{2}\right)+\mathrm{P}^{\max }\left(a_{1}\right) \leq 10 . \\
& -(\mathrm{C} 2 \mathrm{a}) t_{3}^{l}-\left(\mathrm{P}^{\max }\left(a_{3}\right)+\mathrm{P}^{\max }\left(a_{2}\right) \geq 7 .\right. \\
& -(\mathrm{C} 2 \mathrm{~b}) t_{3}^{u}-\left(\mathrm{P}^{\min }\left(a_{3}\right)+\mathrm{P}^{\min }\left(a_{2}\right) \leq 10 .\right. \\
& -(\mathrm{C} 3 \mathrm{a}) t_{2}^{l}+t_{3}^{l}-\left(\mathrm{P}^{\max }\left(a_{3}\right)-\mathrm{P}^{\min }\left(a_{1}\right) \geq 9 .\right. \\
& -(\mathrm{C} 3 \mathrm{~b}) t_{2}^{u}+t_{3}^{u}-\left(\mathrm{P}^{\min }\left(a_{3}\right)-\mathrm{P}^{\max }\left(a_{1}\right)\right) \leq 20 . \\
& -(\mathrm{C} 4) t_{1}^{l} \geq 0 \wedge t_{1}^{u} \geq 0 \wedge t_{2}^{l} \geq 0 \wedge t_{2}^{u} \geq 0 \wedge t_{3}^{l} \geq 0 \wedge t_{3}^{u} \geq 0 . \\
& -(\mathrm{C} 5) t_{1}^{l} \leq t_{1}^{u} \wedge t_{2}^{l} \leq t_{2}^{u} \wedge t_{3}^{l} \leq t_{3}^{u} .
\end{aligned}
$$


(a) Alternative pattern

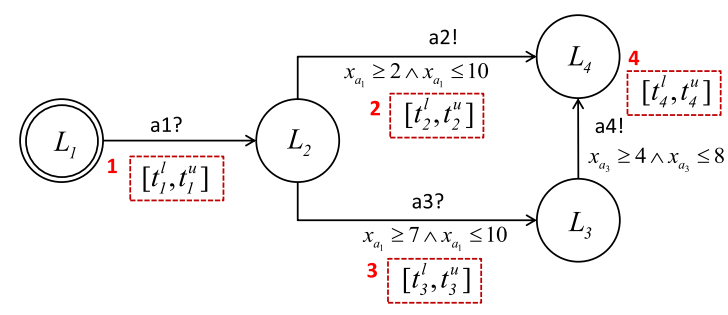

(b) Cyclic pattern

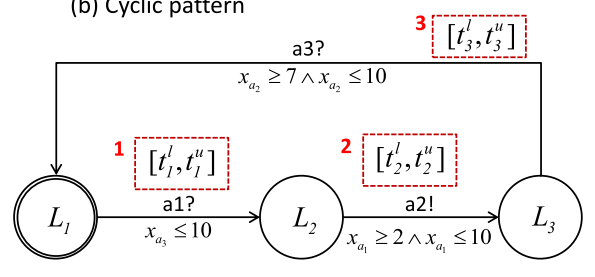

Fig. 13. The example of the variable assignment (Alternative and Cyclic Pattern).

Suppose a platform is characterized as $\mathrm{P}=[1,2]$, and the code is assumed to be generated from the model. In this case, the linear constraints are obtained by plugging in $\mathrm{P}$ as follows: (C1a) $t_{2}^{l} \geq 0$; (C1b) $t_{2}^{u} \leq 6$; (C2a) $t_{3}^{l} \geq 11$; (C2b) $t_{3}^{u} \leq 12$; (C3a) $t_{2}^{l} \geq 10$; (C3b) $t_{2}^{u}+t_{3}^{u} \leq 19$; (C4) $t_{1}^{l} \geq 0 \wedge t_{1}^{u} \geq 0 \wedge$ $t_{2}^{l} \geq 0 \wedge t_{2}^{u} \geq 0 \wedge t_{3}^{l} \geq 0 \wedge t_{3}^{u} \geq 0$; (C5) $t_{1}^{l} \leq t_{1}^{u} \wedge t_{2}^{l} \leq t_{2}^{u} \wedge t_{3}^{l} \leq t_{3}^{u}$.

We explained how linear constraints are formulated with a sequential pattern example. Note that our approach is applicable to a more complex timed automata structure. Figure 13 is the example of alternative and cyclic patterns. In the alternative pattern, there are two different paths. This implies there can be more than one path in between two transition occurrences. In this case, the path that has the minimum delay can be different from the one that has the maximum delay. Therefore, we need to examine all possible paths between the two transitions in order to compute $f_{M}$. In the cyclic pattern, there may exist infinitely many paths. Since we assume non-negative guard conditions, the minimum delay bound is obtained by considering only a simple path. However, when it comes to the maximum delay bound, it differs depending on how many cycles are taken between the two transitions. For example, in the cyclic model of Figure 13, the maximum delay bound between transitions 1 and 3 increases as more cycles are taken. Under a certain restricted timed automata structure, the delay bounds for all pairs of transitions can be formulated by considering only simple paths. More details on the extension can be found in our prior work (Kim et al. 2015b).

\subsection{Formulation of the Optimization Constraints}

As illustrated in Figure 11, the reduction of the code-level delay (3) results in the reduction of the $m c$-delay (4). There can be multiple parameter assignments to reduce the code-level delay given the same constraints. Some parameter assignment may require the code-level delay to be reduced more than necessary preventing the code from performing complex computations such as solving complex matrix equations to compute the control output. Suppose there existtwo parameter assignments that reduce the code-level delay by $100 \mathrm{~ms}$ and $300 \mathrm{~ms}$, respectively. If we choose the assignment that reduces $300 \mathrm{~ms}$, it will give less room for the computation of the code by $200 \mathrm{~ms}$ compared to the choice of $100 \mathrm{~ms}$ reduction. We can introduce the optimization objective in order not to reduce the code-level delay more than necessary. We give one example optimization objective to demonstrate how to find the optimal parameter assignment in terms of the code-level delay. For a pair of $\mathrm{I} / \mathrm{O}$, indexed as $i$, consider its $m c$-delay $\Delta_{\mathrm{IMP}}(i)$. The following optimization objective maximizes the summation of the the $m c$-delay for every pair of $\mathrm{I} / \mathrm{O}$ :

$$
\text { maximize } \sum_{i=1}^{n} \Delta_{\mathrm{IMP}}(i) \text {. }
$$

The $m c$-delay of a pair of $\mathrm{I} / \mathrm{O}$ is characterized by subtracting the minimum delay bound from the maximum delay bound of the occurrence of the second event. For example, the $m c$-delay for 
every pair of I/O event of the model in Figure 12 can be characterized as follows:

$$
\begin{aligned}
\Delta_{\mathrm{IMP}}(1,2)= & \left(t_{2}^{u}+P^{\max }\left(a_{2}\right)+P^{\max }\left(a_{1}\right)\right) \\
& -\left(t_{2}^{l}+P^{\min }\left(a_{2}\right)+P^{\min }\left(a_{1}\right)\right), \\
\Delta_{\mathrm{IMP}}(2,3)= & \left(t_{3}^{u}-\left(P^{\min }\left(a_{3}\right)+P^{\min }\left(a_{2}\right)\right)\right) \\
& -\left(t_{3}^{l}-\left(P^{\max }\left(a_{3}\right)+P^{\max }\left(a_{2}\right)\right)\right), \\
\Delta_{\mathrm{IMP}}(1,3)= & \left(t_{2}^{u}+t_{3}^{u}-\left(P^{\min }\left(a_{3}\right)-P^{\max }\left(a_{1}\right)\right)\right) \\
& -\left(t_{2}^{l}+t_{3}^{l}-\left(P^{\max }\left(a_{3}\right)-P^{\min }\left(a_{1}\right)\right)\right) .
\end{aligned}
$$

Then, the following optimization objective will find the parameter assignment for the code-level delay in a way that maximizes the sum of the $m c$-delays:

$$
\text { maximize } \quad \Delta_{\mathrm{IMP}}(1,2)+\Delta_{\mathrm{IMP}}(2,3)+\Delta_{\mathrm{IMP}}(1,3) .
$$

Note that there can be various forms of the objective functions possible depending on the types of code-level computation for each I/O pair. For example, one can consider a weighted optimization objective to assign more computation time to perform a particular pair of I/O compared to other I/O. It is out of the scope of this article to define all possible forms of the optimization objective.

The formalization introduced in Section 5 enables us to automatically find the new timing parameter assignments that meet the delay-bound inclusion constraint using the ILP solver. If we can enumerate all paths in between each pair of $\mathrm{I} / \mathrm{O}$, the delay-bound inclusion constraints $f_{\mathrm{IMP}}(i, j)$ $\subset f_{M}(i, j)$ can be formulated as well. Then, the solver can find the new timing parameter assignment for the code generation. However, it is not possible to enumerate all possible paths in the presence of a cycle in between the pair of I/O. The following theorem shows that it is sufficient to show the delay-bound inclusion constraints only for the simple path between such $\mathrm{I} / \mathrm{O}$ pair (i.e., the delay-bound inclusion constraint will hold for all paths in between the I/O pair).

Theorem 5.2. Given a platform processing delay $\mathrm{P}$ and a platform-independent model $\mathrm{M}$, if $f_{\mathrm{IMP}}(i, j) \subset f_{M}(i, j)$ for every pair of transitions $t_{i}$ and $t_{j}$, then $f_{\mathrm{IMP}}(p) \subset f_{M}(p)$ for any path $p$ that starts with $t_{i}$ and ends with $t_{j}$.

Proof. Refer to our prior work (Kim et al. 2015b).

\section{THE CASE STUDY}

The main purpose of our case study is to show two aspects:

(1) how much complex platform-independent models can be handled using our framework;

(2) how well the code generated with the original and the adjusted timing parameters from the platform-independent model conform to the delay-bound inclusion constraints.

For purpose (1), we randomly generated 12 different sizes of platform-independent models from the sequential pattern. The model sizes are given in Figure 14 (the blue bar) in terms of the number of transitions (the number of transitions ranges from 5 to 500). Each transition guard is generated from a normal distribution. Each model is associated with a number of unknown $\mathrm{min} / \mathrm{max}$ variables shown in the red bar (the number of variables ranges from 10 to 1,000). In addition, the constraints are generated and their numbers range from 35 to 251,000 shown in the green bar (note that the number of generated constraints exponentially grows to the number of variables). The platform delays used for each constraint are also generated from another normal distribution.

For each model size, we generated 10 models with randomly selected guard conditions and platform delays from normal distributions; we measured the execution time to obtain the 10 optimal 


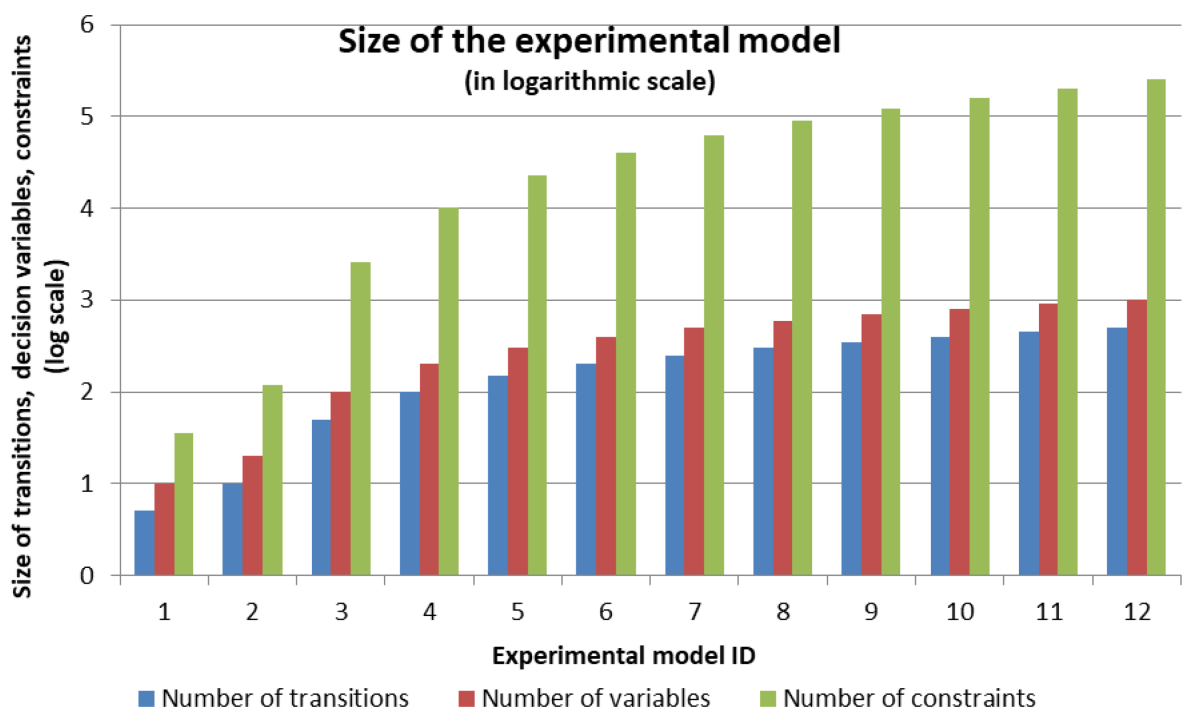

Fig. 14. Model sizes used to measure the execution time to obtain optimal solutions.

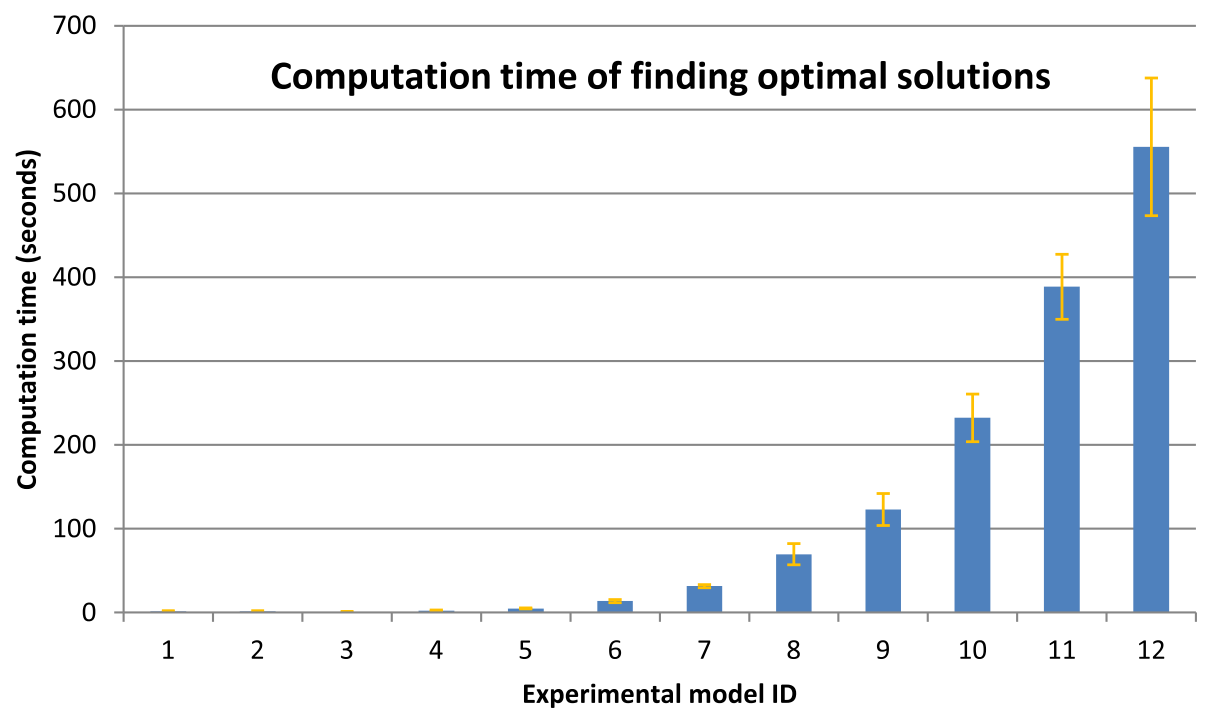

Fig. 15. Computation time required to find optimal solutions.

solutions using the MATLAB linear programming solver. Figure 15 shows the average and the standard deviation of the execution times for each model size. With the size of less than 500 transitions, the solution could be obtained within 15 minutes on the experiment machine (note that this execution time excludes the model generation time). The experimental result also shows the computation time grows exponentially as the model size grows. It is difficult to estimate the execution time precisely in general cases since the execution time may vary depending on the model structures and dependencies among different parameters such as guard conditions and platform delays. However, we believe that this result can be used to roughly assess the amount of overhead needed to determine the timing guards for the code generation purpose using our framework. 


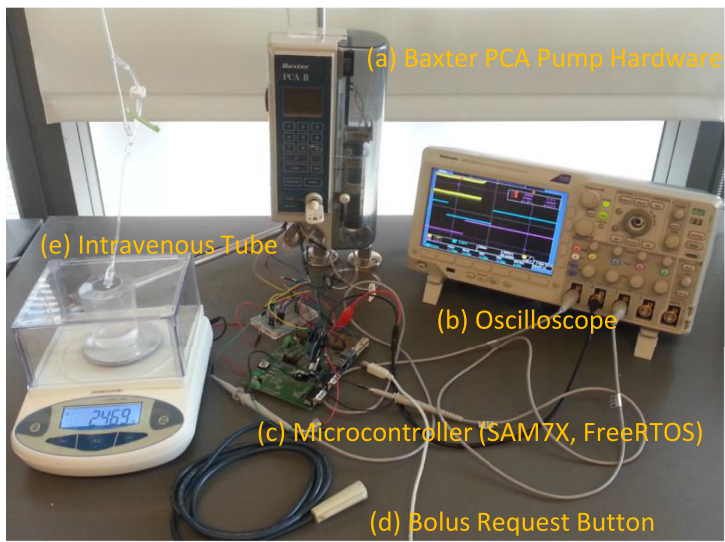

(a) the experimental platform setup

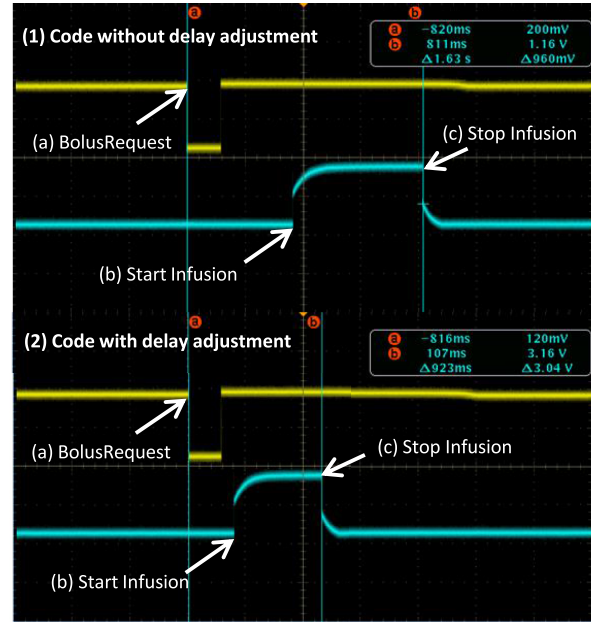

(b) the delay-bound measurement using the oscilloscope

Fig. 16. (a) Case study platform setup (Baxter PCA infusion pump). (b) Delay measurement using the oscilloscope for validation.

For purpose (2), we performed the empirical validation on the PCA infusion pump platform shown in Figure 16 that has been used for the generic PCA infusion pump reference implementation (Kim et al. 2011; Lee et al. 2012). The PCA infusion pump is equipped with the bolus request button that allows the patients to request for an additional amount of drugs whenever they feel pain. Upon pressing a button, a certain amount of drug, called a bolus, flows from the syringe to the patient through the intravenous tube.

The typical safety requirements of PCA infusion pumps (Food and Administration 2009) involves the input and output timing requirement. The following timing requirements are considered by specifying the timing parameters for the case study purpose:

-(REQ1) When a patient requests a bolus, the bolus infusion should start in between 150ms and $470 \mathrm{~ms}$.

-(REQ2) The bolus infusion should be active at least 300ms, and at most $750 \mathrm{~ms}$.

-(REQ3) When a patient requests a bolus, the bolus infusion should finish in between $450 \mathrm{~ms}$ and $1,220 \mathrm{~ms}$ in the absence of the empty reservoir alarm.

We created the platform-independent model to capture the requirements using timed automata. The platform delays were measured for each I/O using the oscilloscope according to our prior test approach (Kim et al. 2014). For example, we measured the delay from the moment a patient presses the button at the mc-boundary until the moment the code is to read the processed input at the io-boundary. We repeated this measurement 20 times from which the min/max platform delays were obtained. The details of the model and the measured platform delays can be found in Kim et al. (2015b). Given the two inputs (i.e., the platform-independent model and the platform delays), we formulated the linear constraints according to the method presented in Section 5 to find the new timing parameters for the code generation. Then, we generated two types of code using the TIMES tool (Amnell et al. 2003): the first code was generated using the same timing parameters of the platform-independent model; the second one was generated using the adjusted timing parameters. Since the tool generates the code using the same timing parameters from the 


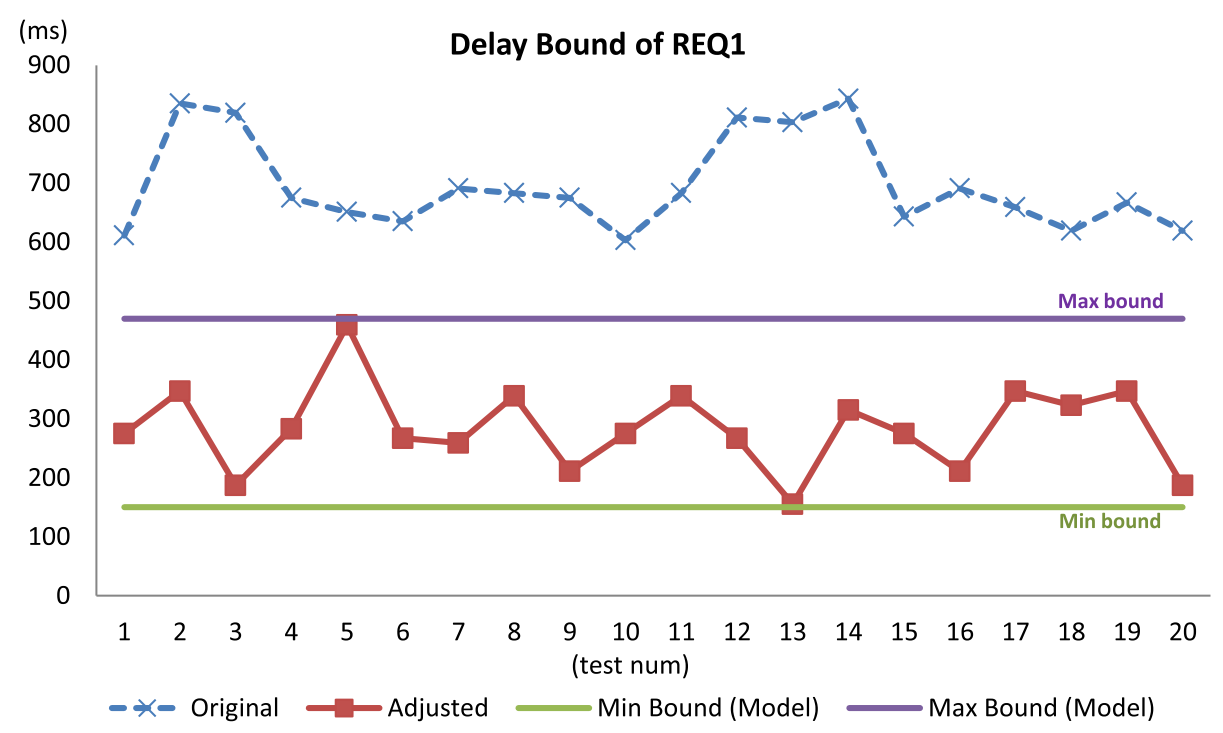

Fig. 17. Validation Result for REQ1.

given model, we only replaced them with the adjusted timing parameters for the second code generation. Each generated code was integrated with the same API to interact with the platform to perform I/O.

Here is the summary of the experiment result to show how the implemented system behaves in terms of the timing requirements. Each requirement specifies its allowed $\mathrm{min} / \mathrm{max}$ delay bound of a pair of I/O. The implemented system is expected to meet this delay bound. In other words, the corresponding $\mathrm{I} / \mathrm{O}$ events occurring at the $m c$-boundary should be separated at least the minimum bound (e.g., 150ms in REQ1) and at most the maximum bound (e.g., 470ms in REQ1) specified in the requirements. The two solid lines in the graphs of Figure 17 indicate such expected $\mathrm{min} / \mathrm{max}$ delay bounds (additional experiment results for REQ2 and REQ3 can be found in Kim et al. (2015b)).

For each implemented system running the code, we measured the actual I/O timing 20 times for each requirement and plotted them in the graphs. The dotted blue lines and the red lines indicate the measured delay between a pair of $\mathrm{I} / \mathrm{O}$ when the system runs the code generated using the original timing parameters and the adjusted timing parameters from the platform-independent model. The experiment results show that the code generated from the adjusted timing parameters always produces the output within the expected delay bound (red lines). On the other hand, the code generated using the original timing parameters does not satisfy the expected delay bound in most cases (blue lines). It is because that the platform delays were not compensated during the code generation so that it is added up to the code-level delay failing to meet the expected delay bound.

\section{RELATED WORK}

There have been several works to incorporate the platform delays to the model. The first model transformation in our work was motivated from Altisen's work (Altisen and Tripakis 2005). This work studied if a timed automata can be implemented on a given platform satisfying a desired property using its standard semantics.

There are two aspects that differentiate our work from Altisen's work. The first aspect is the scope of the possible interaction mechanisms among the code, the platform, and the environment. 
It is important to identify such a scope as it also determines the scope of possible integration scenarios where the proposed framework can be applied. We aim at identifying general interaction types among the three components (i.e., the code, the platform, and the environment) via categorization at the $m c$-boundary interactions (Table 1) and the io-boundary interactions (Table 2). With this categorization, a wide range of platform models can be more systematically constructed as shown by the transformation algorithm in the Appendix. To the best of our knowledge, Altisen's work also introduced a few common situations of such interactions as examples, but does not detail what other options are available and how the platform should be modeled. As a result, there are some common situations not discussed in their work, while we believe it is important. For example, we consider the queuing scheme for the communication between a platform and a code, the polling scheme for reading environmental signals, which are commonly used implementation schemes; however, their work only considered the communication scheme using a shared variable, and the interrupt-based scheme in reading inputs from the environment. In terms of the completeness in identifying the common integration scenarios, our work handles a wider range of scenarios compared to Altisen's work.

The second aspect is the capability of adjusting the timing parameters for the code generation purpose. When Altisen's work finds a property is not satisfied due to the overhead incurred by the platform, there is no further process to adjust the timing parameters for the code generation. However, if the code can be running with a smaller execution time to compensate the platform delays, there may be cases, but not all the times, where the system can meet the property. Our second transformation allows one to identify the amount of time that needs to be reduced to compensate the platform delays.

Sifakis's works also studied how platform-specific timing aspects can be combined with the platform-independent model (Abdellatif et al. 2010; Triki et al. 2013). These works introduced how an abstract model (that is matched to our platform-independent model) can be transformed into a physical model (that is matched to our platform-specific model). The basic idea of their transformation is to construct the physical model by assigning the execution time to each action transition of the abstract model (i.e., transitions that are associated with input or output synchronization); more specifically, a single transition in the abstract model is decomposed into two separate transitions in the physical model; at the first transition, the clocks used in the abstract model freeze while performing the synchronization (i.e., time does not flow while performing synchronizations); then, at the second transition, it compensates the execution time of input (output) by adding timing guards associated with additional clock variables, which enables the transition to be completed after a constant execution time. However, this transformation simplifies several platform-specific aspects that our work wants to focus on. To the best of our knowledge, for example, their transformation does not consider a notion of event queuing or invocation mechanism. Hence, the following scenarios cannot be captured and verified in their physical model: (1) "even though an input event is read by platform, the input may not lead to a transition in the model due to queue overflow condition; (2) "when the generated code is invoked too slow while the frequency of input generated from the environment is relatively faster, the code may not process all the inputs."

An approach was proposed in Wulf et al. (2004) to tackle the timed automata implementability problem based on a new semantics called Almost ASAP that is parameterized by a delay $\Delta$. It presented a way to check whether a model that is constructed using this semantics can be implemented with a sufficiently fast platform; and if a faster platform replaces a slower one, the property still holds. However, this work did not consider what constitutes the platform-specific delays $\Delta$ specifically, whereas ours focuses on the source of timing delay affecting $\Delta$.

Task automata was proposed to extend the timed automata to allow the schedulability analysis of computation tasks (Fersman et al. 2007). A task is characterized as a best/worst execution 
time and a deadline; these timing parameters are associated to a location that can be triggered by events. Triggered tasks are placed into a task queue that processes tasks according to some scheduling strategy (e.g., fixed priority scheduling or earliest deadline first); it shows that a schedulability checking problem can be transformed to a reachability problem. Even though task automata can be also used to express some platform-specific aspects, we characterize a platform from a different perspective; that is, how an input is generated from the environment (e.g., pulse signal or sustained signal), and how an input is accepted by a platform (e.g., interrupt-based or sampling-based mechanism), and how the input (output) is delivered to the code (to the platform) (e.g., shared variables or queues). It is non-trivial to express such details using task automata. However, we believe our work and task automata can complement each other if the two different levels of the platform characterization can be interfaced with each other.

Dellabani et al. proposed a way to schedule a local planning in the multiparty interactions (Dellabani et al. 2016). This work expresses each distributed component as timed automata that may synchronize on particular actions to coordinate their activities. They considered communication delays to the interaction corresponding to the exchange of messages. Their goal is to develop a way to let components be notified ahead through communication primitives in a way they can perform local planning ahead by avoiding a deadlock condition. One of the major outcomes is to show the sufficient condition to ensure a deadlock-free planning of such interactions. Our work is similar to this work in a sense to find a way to desynchronize a certain component (a code in our case and a distributed component in their case) due to a certain constraint (a platform delay in our case and a communication delay in their case) by meeting a certain property (a delay-bound inclusion property in our case and a deadlock-free in their case). Due to the difference in the property of interest, they did not distinguish the impact of input/output interaction on the desynchronization, while ours does. However, we believe that their approach requires less restrictive semantics compared to ours so their work may complement our work in extending the scope of models.

\section{CONCLUSIONS}

We presented a framework to determine the timing parameters of the code generated from the platform-independent model by taking into account the platform delays. The first transformation extends the platform-independent model into the platform-specific model by explicitly modeling the platform aspects such as $\mathrm{I} / \mathrm{O}$ processing and the code invocation mechanism. The resulting platform-specific model is used to verify the delay bounds of the implemented system that runs the code generated with the original timing parameters of the platform-independent model. Such an implemented system may or may not verify the timing requirements since the platform-delay is added up to the code-level delay. The second transformation aims at modifying the original timing parameters to adjust the code-level delay in a way that the implemented system meets the timing requirements. This adjustment mechanism is formulated using integer linear programming in order to determine the unknown $\mathrm{min} / \mathrm{max} \mathrm{I} / \mathrm{O}$ timing of the code. Given a platform, this formulation allows us to check if the adjustment of the code-level delay is feasible or not; if feasible, the solver returns the new timing parameters of the code that will guarantee that the implemented system conforms to the timing requirement. To the best of our knowledge, existing code generators syntactically map the timing parameters of the model to the code, which may violate the verified timing requirements when it is integrated with the platform that introduces another level of delays. Our framework can be easily incorporated as a backend of such existing code generators to guarantee the timing requirement conformance. We also believe that the proposed transformation techniques can be integrated with our prior work (Kim et al. 2013) to generate a broad scope of code from models that have different expressiveness. 


\section{APPENDIX}

\section{A THE TRANSFORMATION ALGORITHM FROM THE PLATFORM-INDEPENDENT MODEL TO THE PLATFORM-SPECIFIC MODEL}

This subsection explains the supplementary information extending Section 4. The transformation from the platform-independent model to the platform-specific model is non-trivial since it needs to take into account new information flows and parameters associated with the categorization of the $m c$-boundary and io-boundary in Table 1 and Table 2. Hence, an ad-hoc transformation may result in incorrect platform-specific models. However, we claim that this process can be systematically performed according to our transformation algorithm. We explain the transformation algorithm for a platform-specific model with an example of the implementation scheme: the Type 1 signal, interrupt read policy at rising edges, queues as a communication mechanism with read all policy, and periodic invocation. Algorithms compatible for other schemes can be designed similarly.

We first define the pseudo operations to explain the algorithm:

- Rename $\left(s_{s r c}, s_{d s t}\right)$ : returns an item whose name is changed from $s_{s r c}$ into $s_{d s t}$.

$-\operatorname{Copy}\left(S_{s r c}, S_{d s t}\right)$ : a pairwise copy of items in a set $S_{s r c}$ into another set $S_{d s t}$.

- Insert $\left(s_{s r c}, S_{d s t}\right)$ : inserts an item $s_{s r c}$ into a set $S_{d s t}$.

- CreateL $\left(l_{k}, i n v_{k}\right)$ : creates a new location $l_{s r c}$ whose invariant is $i n v_{k}$.

- CreateT $\left(e, l_{s r c}, l_{d s t}\right)$ : creates a new transition $e$ whose source location is $l_{s r c}$, and destination location is $l_{d s t}$.

- Associate $(e, g, a, u)$ : Associate a transition $e$ with a guard condition $(g)$, and an input or an output synchronization $(a)$, and an update statement $(u)$.

$-I D(s)$ : returns an identification of $s$, which is either a location or a synchronization.

(1) Construction of $\boldsymbol{M}_{\boldsymbol{i o}}: M_{I O}$ is defined as $M_{I O}=\left(L_{I O}, l_{I O 0}, C_{I O}, A_{I O}, E_{I O}, I_{I O}\right)$, and Listing 1 is a pseudo algorithm (Transform 1) that constructs $M_{i o}$ from $M$. Intuitively, $M_{i o}$ remains syntactically the same as $M$, except that its synchronizations are renamed from $m$ to $i$, and from $c$ to $o$. curLoc is an integer variable that keeps track of current locations of $M_{i o}$; this variable is updated every transition to record the identification of the current location. The value of curLoc is used to perform necessary synchronizations in $E X E_{i o}$ that will be introduced in Subsection (3).

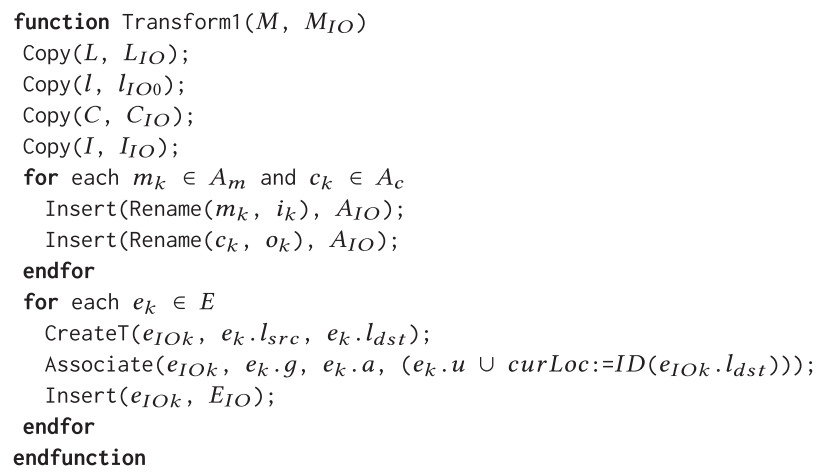

Listing 1. The pseudo algorithm for $M$ to $M_{I O}$ transformation.

In contrast, the environment model $E N V_{m c}$ remains exactly the same under the current implementation scheme. Figure 18-(1) is the example of $M_{i o}$ transformed from $M$ in Figure 1-(1) using the algorithm Transform 1; Figure 18-(2) is the example of $E N V_{m c}$ transformed from $E N V$ in Figure 1-(2). 


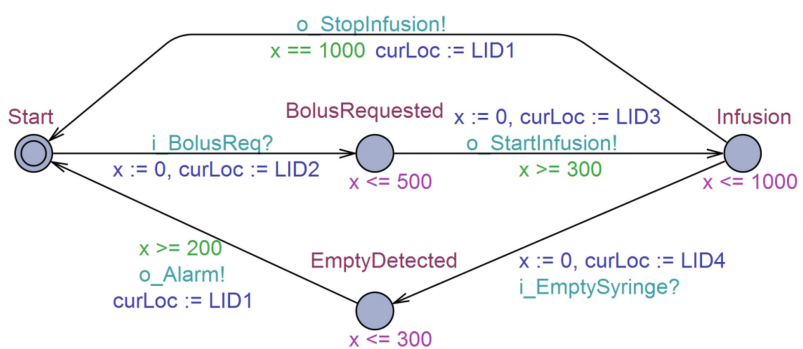

(1) Mı

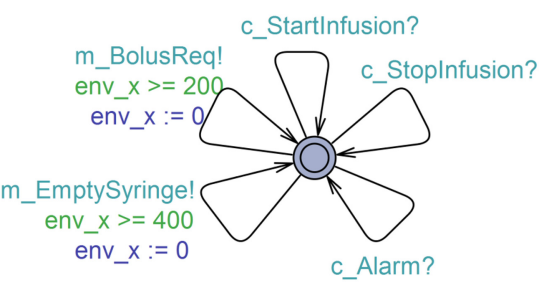

(2) ENVMC

Fig. 18. The $M_{I O}$ and $E N V_{M C}$ of the platform-specific model.

(2) Construction of $I F_{m i}$ and $I F_{o c}: I F_{M I}$ is a network of UPPAAL automata defined as $I F_{M I}=$ $I F_{M I_{1}}\left\|I F_{M I_{2}}\right\| \ldots \| I F_{M I_{k}}$, where $k$ is the number of input synchronizations in $A_{m}$ of $M$. An $I F_{M I_{k}}$ automaton is defined as $I F_{M I_{k}}=\left(L_{M I}, l_{M I_{0}}, C_{M I}, A_{M I}, E_{M I}, I_{M I}\right)$. Listing 2 is the pseudo algorithm (Transform2) that constructs $I F_{m i}$ from $M$ ( $I S_{1}$ refers to the chosen implementation scheme).

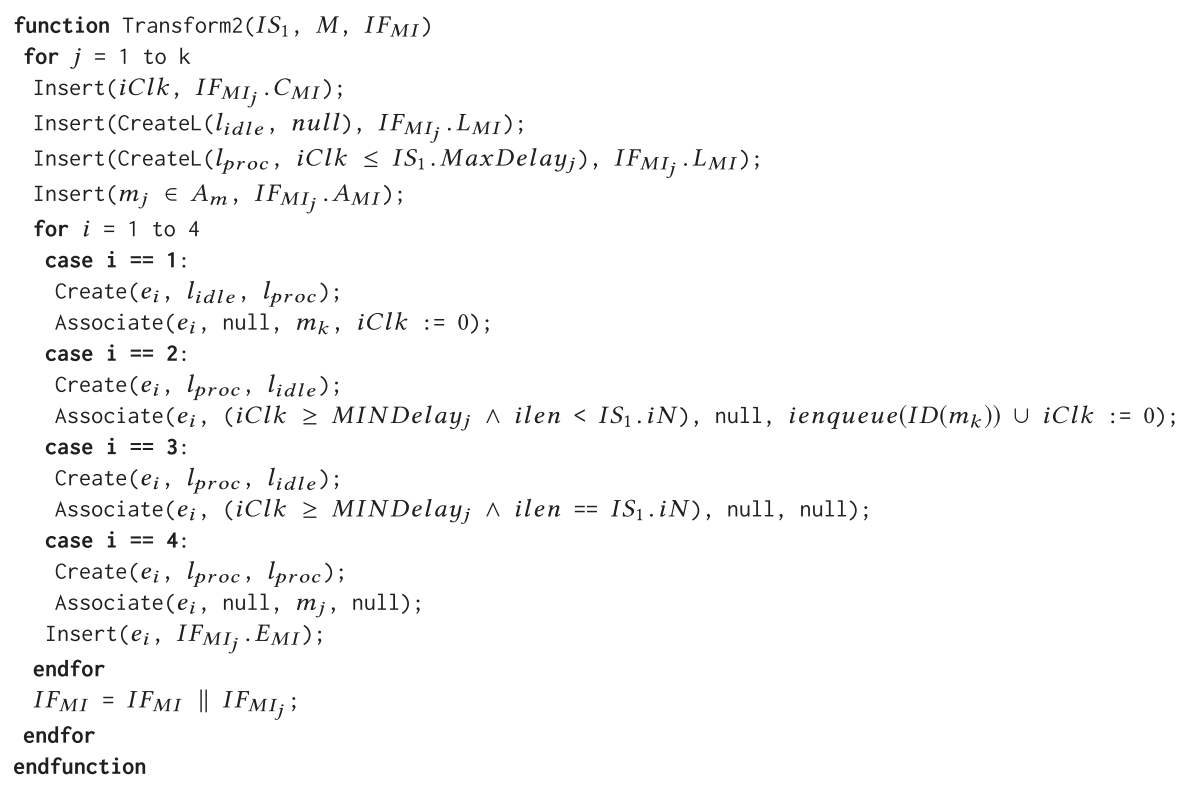

Listing 2. The pseudo algorithm for $I F_{M I}$ construction.

Figure 19-(1) and Figure 19-(2) show the automaton $I F_{M I_{\text {BolusReq }}}$ and $I F_{M I_{\text {Emptysyringe }}}$ constructed from $M$ in Figure 1 and $I S_{1}$. At the location Idle, the Input-Device is ready to read an input $m_{j}$ from $E N V_{m c}$. At the location Processing, the Input-Device is currently processing the input $m_{j}$ that has been read from $E N V_{m c}$. Once the input $m_{j}$ is read (when a transition from Idle to Processing is taken), a

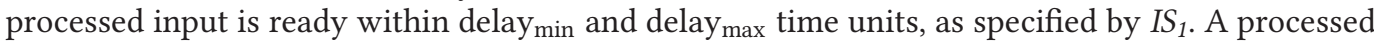
input is delivered to the software through a finite buffer whose size is defined in the parameter buffer-size. Therefore, there are two cases when the processed input needs to be inserted into the buffer: (1) the buffer has an empty slot, and (2) the buffer is full. These two cases are modeled as the two respective transitions from Processing to Idle. 


\section{(1) IFMI_EmptySyringe}

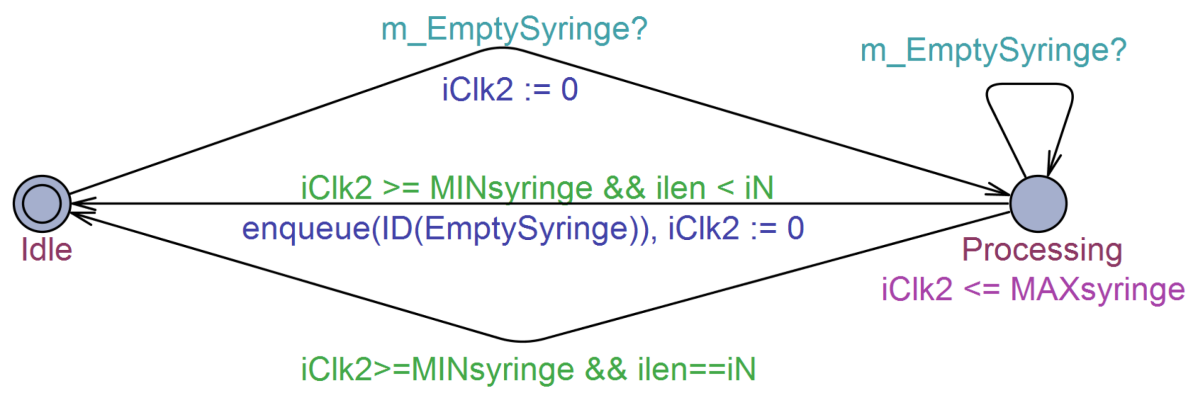

(2) IFoc_StopInfusion

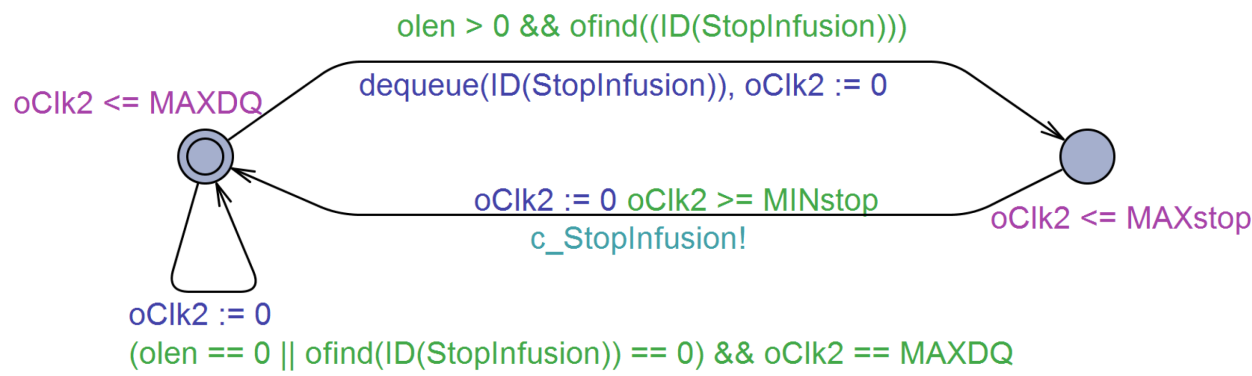

(3) IFoc_Alarm

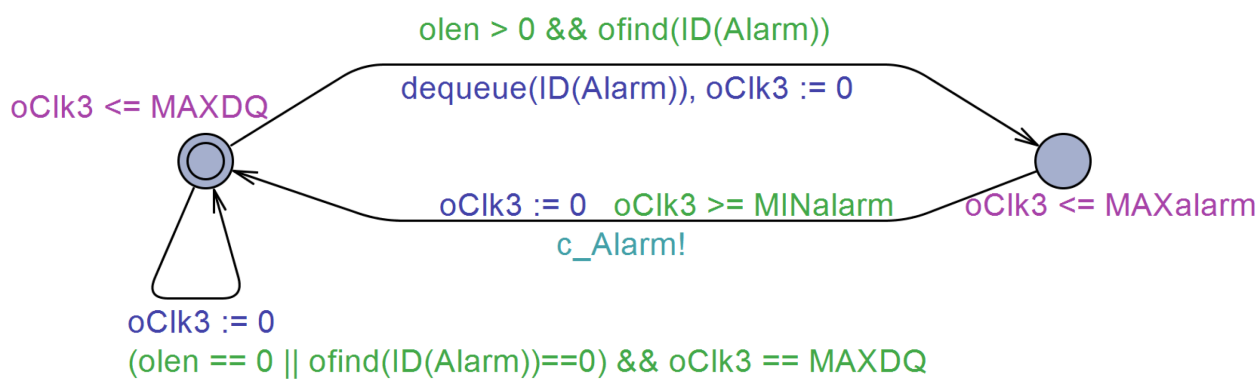

Fig. 19. The $I F_{M I-E m p t y S y r i n g e}$ and $I F_{O C-S t o p l n f u s i o n}$ and $I F_{O C-A l a r m}$ of PSM.

$I F_{o c}$ is an output interface automata that abstracts the dataflows from $o$ to $c$ that are performed by the Output-Device in Figure 6. $I F_{o c}$ is synchronized with $E N V_{m c}$ over c-variables to read outputs from the software. Then, it finishes output processing in between minimum and maximum processing delay defined in $I S_{1}$. The processed outputs in the form of $c$-variables are ready to be written to $E N V_{m c}$. The purpose of this transformation is to obtain $I F_{o c}$ that abstracts such timing behavior of the Output-Device. The detailed construction algorithm is shown in Listing 3 (the construction is similar to Listing 2). Figure 19-(2) and (3) are the examples $I F_{O C_{\text {Stopinfusion }}}$ and $I F_{O C_{\text {Alarm }}}$ transformed from $M$ in Figure 1 and $I S_{1}$.

(3) Construction of $E X E_{i o}: E X E_{i o}$ is an UPPAAL automaton that is defined as $E X E_{i o}=\left(L_{E X E}, l_{E X E 0}\right.$, $\left.C_{E X E}, A_{E X E}, E_{E X E}, I_{E X E}\right)$. The construction of $E X E_{i o}$ from $M_{i o}$ and $I S_{1}$ consists of the construction for the non-buffer operations and the buffer operations. 


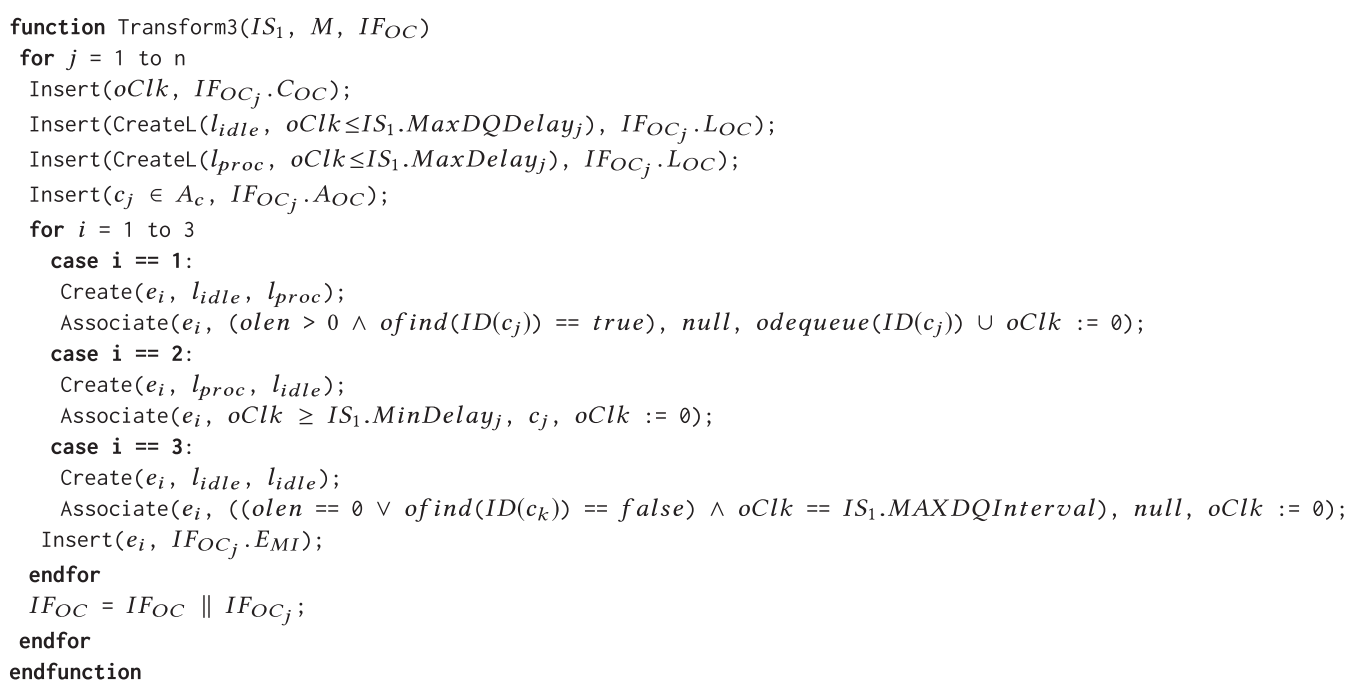

Listing 3. The pseudo algorithm for $M$ to $I F_{O C}$ transformation.

The non-buffer operation part in Listing 4 is the algorithm that constructs a part of $E X E_{i o}$. It creates six locations that correspond to the execution stages of the code execution as follows: Waiting indicates that the code is waiting for an invocation; Active location indicates that the code has been invoked and is ready for execution; ReadReady, ReadComplete, WriteReady, WriteComplete locations indicate the respective computation stages-read input, compute transitions, write output-are being performed. The two platform-specific parameters, InvokePrd and WCET, are associated with the invariants of these locations. InvokePrd is the invariants of Waiting that specify the periodic invocation interval; WCET is the invariant of the rest of the locations that specifies the worst-case execution time of the code. Figure 10 is the $E X E_{i o}$ constructed from $M_{i o}$ in Figure 18-(1) where these six locations are represented.

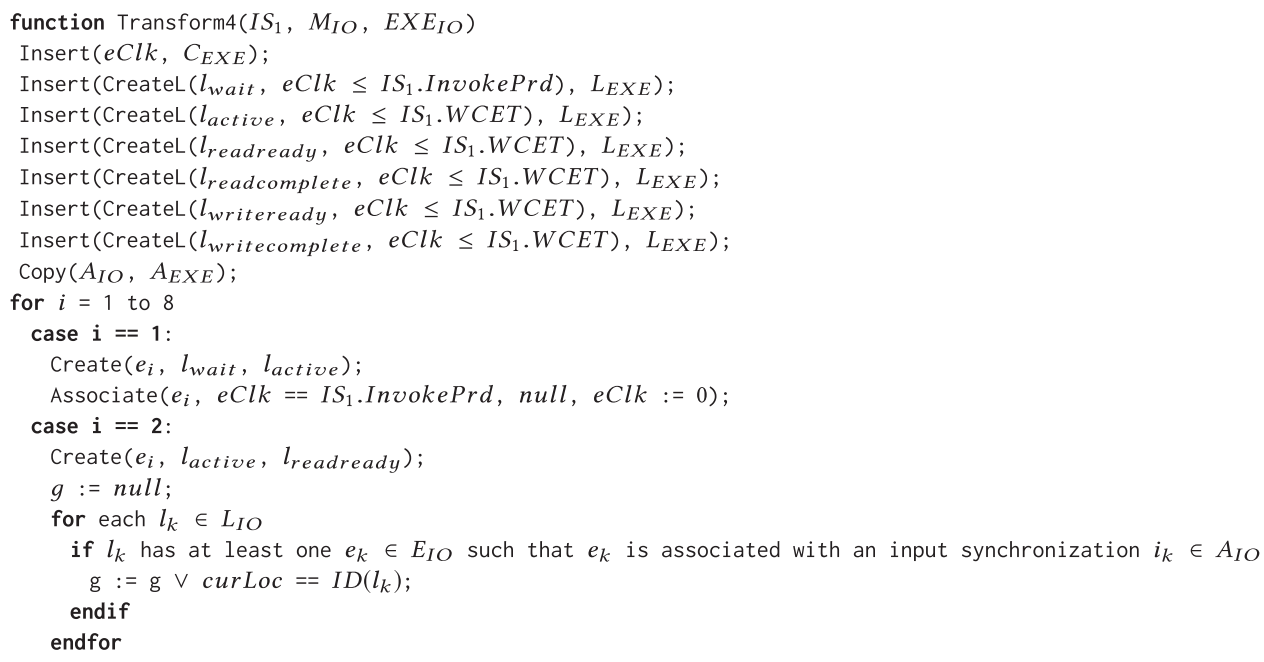




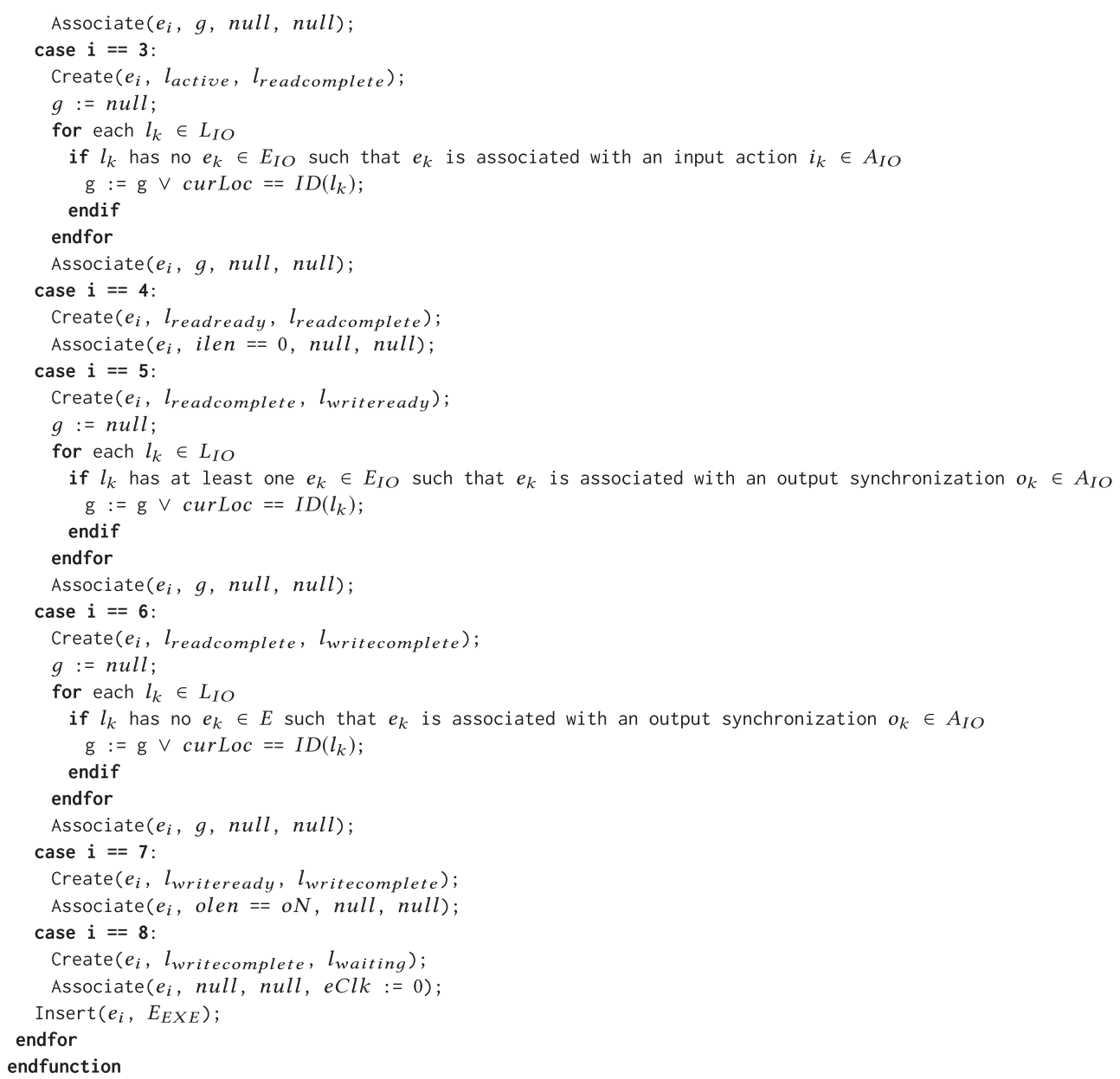

Listing 4. The pseudo algorithm for construction $E X E_{I O}$ from $M_{I O}$ to transformation (Non-buffer operation part).

Listing 5 is the transformation algorithm that constructs the buffer operation part of $E X E_{i o}$. Given the constructed locations using the non-buffer operation part, this algorithm basically adds necessary input and output transitions according to the following rules. For an input (output) synchronization associated with a transition in $M_{i o}$, it creates two types of transitions from ReadReady (WriteReady) to ReadComplete (WriteComplete) in EXE $E_{i o}$ and associates it with the conjunction of three guard conditions: (1) $M_{i o}$ is in a location that can read the input (write the output), and (2) the original guard condition of the transition in $M_{i o}$, and (3) the input is in the buffer (the output buffer is not full). One transition indicates that there is an input in the buffer (the output buffer is not full), and the associated guard condition of the transition in $M_{i o}$ is satisfied; then, it dequeues the input performing the input synchronization (enqueues the output to the buffer performing the output synchronization) between $M_{i o}$ and $E X E_{i o}$. The other transition indicates that the associated guard condition of the transition in $M_{i o}$ is not satisfied, or there is no input in the buffer (the output buffer is full); in this case, it cannot perform the respective synchronization. 


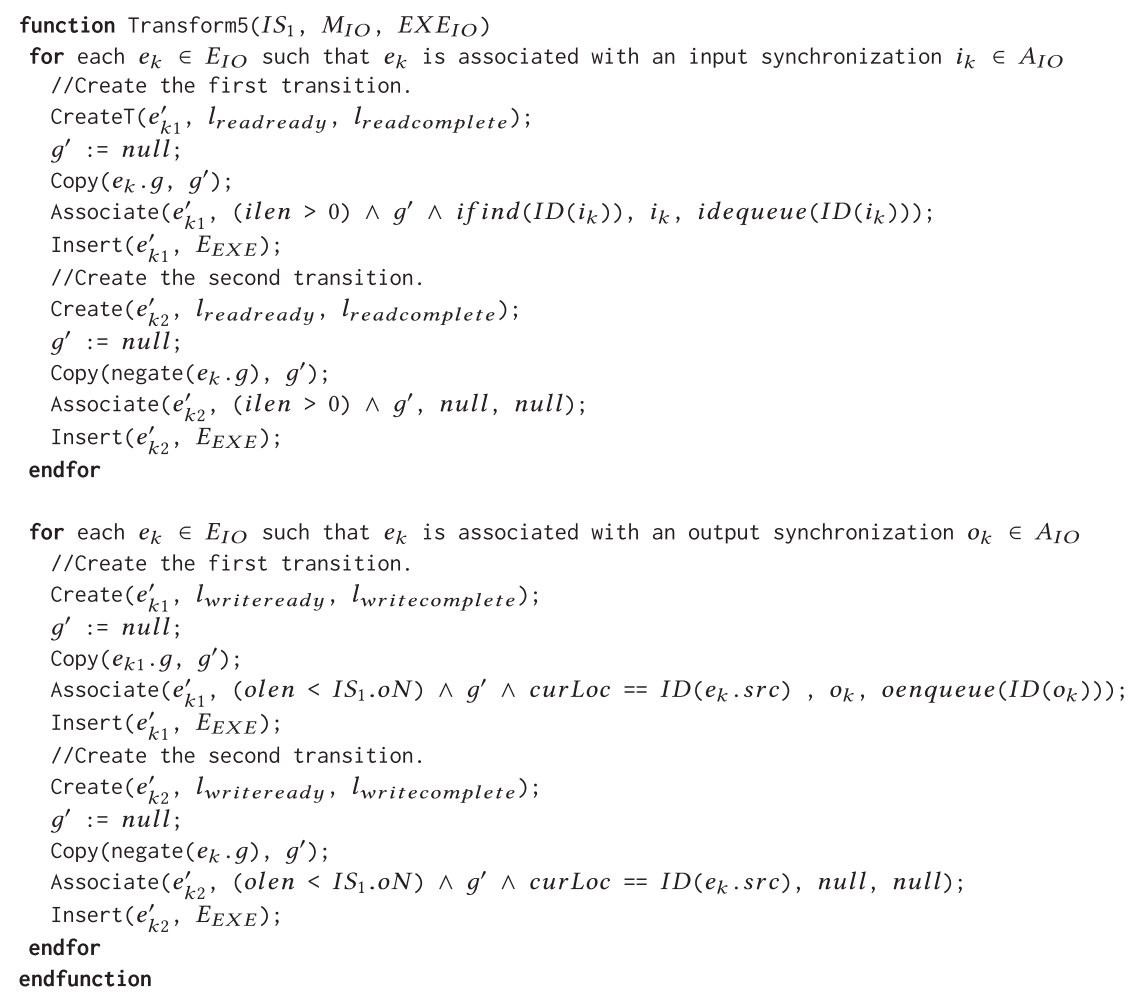

Listing 5. The pseudo algorithm for construction $E X E_{I O}$ from $M_{I O}$ (Buffer-operation part).

\section{REFERENCES}

Y. Abdeddaïm, E. Asarin, and O. Maler. 2006. Scheduling with timed automata. Theoretical Computer Science 354, 2 (2006), $272-300$

Tesnim Abdellatif, Jacques Combaz, and Joseph Sifakis. 2010. Model-based implementation of real-time applications. In EMSOFT. ACM.

K. Altisen and S. Tripakis. 2005. Implementation of timed automata: An issue of semantics or modeling. FORMATS (2005).

Rajeev Alur and David L. Dill. 1994. A theory of timed automata. Theoretical Computer Science 126, 2 (1994), 183-235. DOI : https://doi.org/10.1016/0304-3975(94)90010-8

Rajeev Alur, Limor Fix, and Thomas A. Henzinger. 1999. Event-clock automata: A determinizable class of timed automata. Theoretical Computer Science 211, 1-2 (1999), 253-273.

Rajeev Alur and Gera Weiss. 2008. RTComposer: A framework for real-time components with scheduling interfaces. In EMSOFT.

T. Amnell, E. Fersman, L. Mokrushin, P. Pettersson, and W. Yi. 2003. TIMES: A tool for schedulability analysis and code generation of real-time systems. In FORMATS.

G. Behrmann, A. David, and K. G. Larsen. 2004. A tutorial on Uppaal. In FORMAT.

Mahieddine Dellabani, Jacques Combaz, Marius Bozga, and Saddek Bensalem. 2016. Local Planning of Multiparty Interactions with Bounded Horizons. Springer International Publishing, Cham, 199-216. DOI:https://doi.org/10.1007/ 978-3-319-48989-6_13

Elena Fersman, Pavel Krčál, Paul Pettersson, and Wang Yi. 2007. Task automata: Schedulability, decidability and undecidability. Information and Computation 205, 8 (2007), 1149-1172. DOI : https://doi.org/10.1016/j.ic.2007.01.009

U.S. Food and Drug Administration. 2009. Safety requirements for the generic patient controlled analgesia pump. http:// rtg.cis.upenn.edu/gip.php3. (2009).

Marc Geilen, Stavros Tripakis, and Maarten Wiggers. 2011. The earlier the better: A theory of timed actor interfaces. In HSCC.

Inhye Kang and Insup Lee. 1996. An efficient state space generation for analysis of real-time systems. In ISSTA'96. 
BaekGyu Kim, Anaheed Ayoub, Oleg Sokolsky, Insup Lee, Paul Jones, Yi Zhang, and Raoul Jetley. 2011. Safety-assured development of the GPCA infusion pump software. In Proceedings of the 9th ACM International Conference on Embedded Software (EMSOFT'11). ACM, New York, NY, 155-164. DOI : https://doi.org/10.1145/2038642.2038667

BaekGyu Kim, Lu Feng, Linh T. X. Phan, Oleg Sokolsky, and Insup Lee. 2015a. Platform-specific timing verification framework in model-based implementation. In DATE'15.

BaekGyu Kim, Lu Feng, Oleg Sokolsky, and Insup Lee. 2015b. Platform-specific code generation from platform-independent timed models. In IEEE Real-Time Systems Symposium (RTSS'15).

B. Kim, H. I. Hwang, T. Park, S. H. Son, and I. Lee. 2014. A layered approach for testing timing in the model-based implementation. In 2014 Design, Automation Test in Europe Conference Exhibition (DATE'14). 1-4. DOI : https://doi.org/10. 7873/DATE.2014.202

B. Kim, L. T. X. Phan, O. Sokolsky, and L. Lee. 2013. Platform-dependent code generation for embedded real-time software. In 2013 International Conference on Compilers, Architecture and Synthesis for Embedded Systems (CASES'13). 1-10. DOI : https://doi.org/10.1109/CASES.2013.6662512

Pavel Krčál, Leonid Mokrushin, P. S. Thiagarajan, and Wang Yi. 2004. Timed vs. time-triggered automata. In CONCUR.

Pavel Krčál and Radek Pelánek. 2005. On sampled semantics of timed systems. In FSTTCS.

I. Lee, O. Sokolsky, S. Chen, J. Hatcliff, E. Jee, B. Kim, A. King, M. Mullen-Fortino, S. Park, A. Roederer, and K. K. Venkatasubramanian. 2012. Challenges and research directions in medical cyber physical systems. Proceedings of the IEEE 100, 1 (Jan. 2012), 75-90. DOI : https://doi.org/10.1109/JPROC.2011.2165270

David Lorge Parnas and Jan Madey. 1995. Functional documents for computer systems. Science of Computer Programming 25 (1995), 41-61.

Ingo Stierand, Philipp Reinkemeier, Tayfun Gezgin, and Purandar Bhaduri. 2013. Real-time scheduling interfaces and contracts for the design of distributed embedded systems. In SIES

Ahlem Triki, Jacques Combaz, Saddek Bensalem, and Joseph Sifakis. 2013. Model-based implementation of parallel realtime systems. In FASE'13. DOI : http://dx.doi.org/10.1007/978-3-642-37057-1_18

Martin Wulf, Laurent Doyen, and Jean-François Raskin. 2004. Almost ASAP semantics: From timed models to timed implementations. In HSCC.

Received October 2017; revised April 2018; accepted May 2018 